\title{
Asymmetric Double Strange Attractors in a Simple Autonomous Jerk Circuit
}

\author{
G. H. Kom $\mathbb{D}^{1},{ }^{1,2}$ J. Kengne, ${ }^{1}$ J. R. Mboupda Pone, ${ }^{1,2}$ G. Kenne $\mathbb{D}^{1},{ }^{1}$ and A. B. Tiedeu ${ }^{2}$ \\ ${ }^{1}$ Laboratoire d'Automatique et Informatique Appliquée (LAIA), Department of Electrical Engineering, IUT-FV Bandjoun, \\ University of Dschang, P.O. Box 134, Bandjoun, Cameroon \\ ${ }^{2}$ Groupe de Recherche sur les Technologies Médicales Adaptées aux Tropiques (GRETMAT), Laboratoire d'Electronique et de \\ Traitement du Signal (LETS), ENSP, University of Yaoundé I, P.O. Box 8390, Yaounde, Cameroon
}

Correspondence should be addressed to G. H. Kom; koguiho2008@yahoo.fr

Received 19 June 2017; Accepted 17 August 2017; Published 8 February 2018

Academic Editor: Mohamed Belhaq

Copyright (C) 2018 G. H. Kom et al. This is an open access article distributed under the Creative Commons Attribution License, which permits unrestricted use, distribution, and reproduction in any medium, provided the original work is properly cited.

The dynamics of a simple autonomous jerk circuit previously introduced by Sprott in 2011 are investigated. In this paper, the model is described by a three-time continuous dimensional autonomous system with an exponential nonlinearity. Using standard nonlinear techniques such as time series, bifurcation diagrams, Lyapunov exponent plots, and Poincare sections, the dynamics of the system are characterized with respect to its parameters. Period-doubling bifurcations, periodic windows, and coexisting bifurcations are reported. As a major result of this work, it is found that the system experiences the unusual phenomenon of asymmetric bistability marked by the presence of two different attractors (e.g., screw-like Shilnikov attractor with a spiralling-like Feigenbaum attractor) for the same parameters setting, depending solely on the choice of initial states. Among few cases of lower dimensional systems capable of such type of behavior reported to date (e.g., Colpitts oscillator, Newton-Leipnik system, and hyperchaotic oscillator with gyrators), the jerk circuit/system considered in this work represents the simplest prototype. Results of theoretical analysis are perfectly reproduced by laboratory experimental measurements.

\section{Introduction}

The phenomenon of multistability (i.e., the occurrence of multiple attractors for the same parameters setting depending solely on the choice of initial conditions) has captivated the attention of most researchers in recent years. They have done many works in various fields of science and engineering such as electrical circuits [1-5], laser systems [6,7], biological systems [8], and chemical reactions [9]. Systems with only one attractor are called monostable systems. In such systems, the basin of attraction (i.e., the set of initial conditions for which the asymptotic dynamics converge to the underlined attractor) is the whole state space. In contrast, in a multistable system, each attractor has its own basin of attraction. Correspondingly, the basin boundaries can have a simple structure (simple demarcation) or a very complex structure (i.e., nontrivial or fractal basin boundaries). A physical implication of fractal basin boundaries is random jump between coexisting attractors in experiment. Various types of attractors can coexist such as fixed points, period-n limit cycles, toruses, and strange attractors. Multistability makes a system offer a great flexibility [10]. In particular, the coexistence of infinitely many attractors is called extreme multistability and has been reported in two unidirectionally coupled Lorenz systems [11], two bidirectionally coupled Rossler oscillators [12, 13], and very recently a memristor oscillator [14]. Multistability can be advantageously exploited for image processing [10] or taken as an additional source of randomness which is particularly suited for information engineering applications [15]. In general, the phenomenon of multiple attractors is mostly observed in symmetric dynamical systems [16]. Such systems exhibit pairs of mutually symmetric attractors that merge to form a single symmetric one via the well-known attractor merging crisis as a parameter is varied. However, asymmetric multistability (i.e., coexistence of nonsymmetric attractors) is also reported in systems without any symmetry such as Colpitts oscillator [3], Newton-Leipnik system [17], and hyperchaotic oscillator with gyrators [4]. 


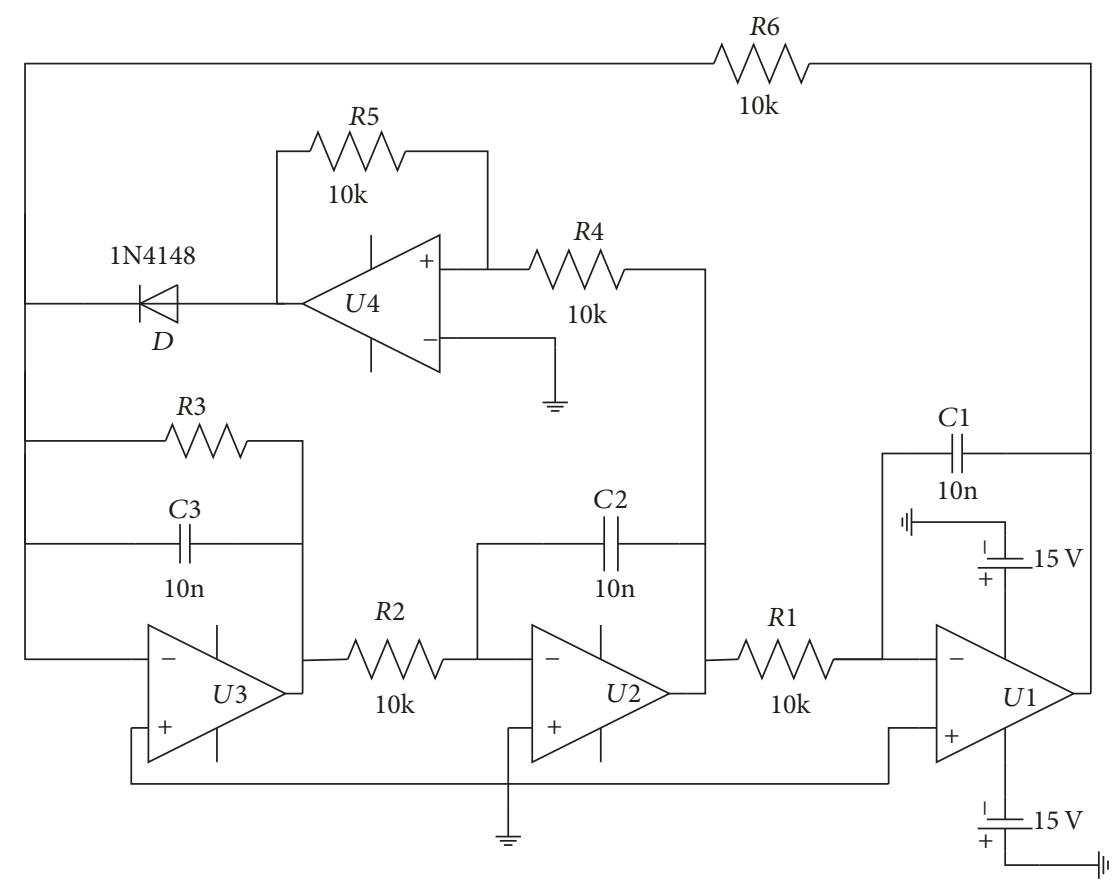

FIGURE 1: Electronic circuit realization of the simple chaotic jerk system under consideration. The simplicity of the circuit is remarkable: only RC components, a diode, and a quadruple op. amplifier (TL084) are involved. The circuit has been built using the sets of element values as shown in the figure. $R_{3}$ is tuneable element used as bifurcation control parameter. $\mathrm{OA}_{1}, \mathrm{OA}_{2}, \mathrm{OA}_{3}$, and $\mathrm{OA}_{4}$ are TL084 type or equivalent operational amplifiers. $D$ is a general purpose diode; here $D=1 \mathrm{~N} 4148\left(\eta=1.9, V_{T}=26 \mathrm{mV}\right.$, and $\left.I_{S}=2.682 \mathrm{nA}\right) . C_{1}=C_{2}=C_{3}=C=10 \mathrm{nF}$; $R_{1}=R_{2}=R_{4}=R_{5}=R_{6}=R=10 \mathrm{k} \Omega$.

In the present contribution, we consider the dynamics of an extremely simple chaotic jerk circuit recently introduced by Sprott [18] with particular attention on the chaos mechanism as well as the possibility of multiple coexisting attractors. In this particular jerk circuit, the exponential nonlinearity is implemented by a single semiconductor diode. Therefore, the model is nonsymmetric and thus generic. Furthermore, the circuit cannot support symmetric attractors. However, as mentioned above, the possibility of multiple coexisting attractors is not excluded. To begin, considering results of [18-20], we recall that jerk systems are third-order differential equations of the form $\dddot{x}=J(\ddot{x}, \dot{x}, x)$. The term $J(\cdot)$ is the nonlinear function and designated the "jerk." It indicates the third-time derivative of $x$ which corresponds to the first-time derivative of acceleration in mechanical system. In the studies undertaken by Eichhorn et al. [21] on multistability behavior of simple asymmetric jerk systems, the authors explore the dynamics of two simplest polynomial jerky dynamics (JD1: $\dddot{x}=k_{1} \ddot{x}+k_{2} x+x \dot{x}+k_{3}$ and JD2: $\left.\ddot{x}=k_{1} \ddot{x}+k_{2} x+x^{2}+k_{3}\right)$ known to experience chaotic behavior in some parameter ranges. The authors, by numerical estimation of Lyapunov spectra, also establish dependence of long-time dynamical behavior on the system parameters. Forward and backward bifurcation diagrams have been used to study some parameters of the dependence on initial conditions (e.g., coexistence of two stable attractors, hysteresis). In both (JD1 and JD2) cases not more than two coexisting attractors are found due to the absence of any symmetry. Very recently, a series of works concerning the issue of coexisting multiple attractors in simple jerk dynamical systems were carried out by Kengne et al. [2, 22]. Motivated by the outcomes we have mentioned above, this paper studies the dynamics of the simple jerk circuit previously introduced by Sprott [18] with the following key objectives: (a) to carry out a systematic analysis of the novel jerk circuit and explain the chaos mechanism; (b) to precise the region in parameter space, in which the proposed model exhibits multiple coexisting attractors and hysteretic dynamics; (c) to realize an experimental study of the system to support the theoretical predictions. More importantly, we provide some design tools (i.e., bifurcation diagrams) that are of precious utility for a practical circuit design of this type of oscillators in relevant engineering applications.

The rest of the paper is organised as follows. Section 2 deals with modeling process. The electronic structure of the novel jerk circuit is described and an appropriate mathematical model is derived. In Section 3 the complex dynamics of the oscillator are investigated from normalized mathematical model. Basic properties of the model are also discussed. The stability of the single equilibrium point is analyzed and conditions for the occurrence of Hopf bifurcation are obtained. In Section 4, the bifurcation structures of the system are investigated numerically showing period-doubling, periodic windows, and coexisting bifurcations. Some windows (in the parameter space) corresponding to the occurrence of multiple coexisting attractors (for the same parameters settings) are uncovered. Correspondingly, basins of attraction of various competing attractors are depicted showing nontrivial basin boundaries. Section 5 is devoted to the laboratory 
experimental study. In this section, an appropriate analog computer is proposed for the investigation of the dynamic behavior of the jerk system. The physical implementation of the oscillator is carried out using electronic components. Laboratory experimental measurements show a very good agreement with the theoretical analysis. Finally some concluding remarks and proposal for future work are presented in Section 6.

\section{Circuit Description and State Equation}

2.1. Circuit Description. Figure 1 shows the electronic structure of the jerk circuit under consideration [18].

The circuit can be decomposed in an oscillating part (linear elements) consisting of three successive integrators in a multiple feedback loop and a nonlinear (velocity) feedback loop involving a negative gain amplifier with a semiconductor diode. The simplicity of the circuit is remarkable: only RC components, a diode, and a quadruple op. amplifier (TL084) are involved. The circuit can be scaled over a wide range of frequencies provided an appropriate choice of capacitors' values. In contrast to the original circuit [18] with equal values components, different values of resistors are introduced to add more control parameters. The values of electronic components are listed in the caption of Figure 1. We would like to stress that the presence of the semiconductor diode (with exponential nonlinearity) is responsible for the complex behavior experienced by the complete electronic circuit. One should also notice that the circuit in Figure 1 is relatively simple compared to the jerk circuit with nonlinear position feedback recently studied by Kengne et al. [2].

2.2. State Equation. In order to derive the state equations for the novel jerk system, some key assumptions are adopted. First, we assume linear capacitors and ideal op. amps operating in their linear working zone. Second, the currentvoltage characteristic $\left(I_{d}-V_{d}\right)$ of the semiconductor diode $(D)$ is obtained from the Shockley diode equation $[23,24]$ as follows:

$$
I_{d}=I_{S}\left(\exp \left(\frac{V_{d}}{\eta V_{T}}\right)-1\right),
$$

where $I_{S}$ is the saturation current of the junction, $V_{T}=k_{b} T / q$ is the thermal voltage with $k_{b}$, the Boltzmann constant, $T$ is the absolute temperature expressed in Kelvin, $q$ is the electron charge, and $\eta$ is the ideality factor $(1<\eta<2)$. With the above simplifying assumptions, using Kirchhoff current and voltage laws, it can be easily shown that the voltages $v_{1}, v_{2}$, and $v_{3}$ satisfy the following set of three coupled first-order nonlinear differential equations:

$$
\begin{aligned}
& \frac{d v_{1}}{d t}=-\frac{v_{2}}{R_{1} C_{1}}, \\
& \frac{d v_{2}}{d t}=-\frac{v_{3}}{R_{2} C_{2}}, \\
& \frac{d v_{3}}{d t}=-\frac{v_{1}}{R_{6} C_{3}}-\frac{v_{3}}{R_{c} C_{3}}-\frac{I_{s}}{C_{3}}\left(\exp \left(\frac{v_{2}}{\eta V_{T}}\right)-1\right),
\end{aligned}
$$

with the adoption of the following change of variables and parameters:

$$
\begin{aligned}
x & =\frac{v_{1}}{V_{\mathrm{Re} f}} ; \\
y & =-\frac{v_{2}}{V_{\mathrm{Re} f}} ; \\
z & =\frac{v_{3}}{V_{\mathrm{Re} f}} ; \\
\mu & =\frac{R}{R_{3}} ; \\
\alpha & =\frac{R}{R_{2}} ; \\
\rho & =\frac{R}{R_{6}} ; \\
\varepsilon & =\frac{R I_{s}}{V_{\mathrm{Re} f}} ; \\
\eta \cdot V_{T} & =V_{\mathrm{Re} f} ; \\
t & =\tau R C .
\end{aligned}
$$

The nondimensional circuit equations are expressed by the following nonlinear third-order differential equations convenient for numerical analysis:

$$
\begin{aligned}
& \dot{x}=y, \\
& \dot{y}=a z, \\
& \dot{z}=-\rho x-\mu z-\varepsilon(\exp (y)-1),
\end{aligned}
$$

where the dot denotes differentiation with respect to the dimensionless time $\tau$. First of all, notice that the vector field (4) is of $C^{\infty}$ (i.e., infinitely differentiable) type due to the presence of the exponential nonlinearity. Also remark that only one state variable, namely, $y$, is involved in the hyperbolic nonlinearity of the model and hence the nonlinear velocity feedback of the system. The presence of this nonlinearity is responsible for the complex behaviors experienced by the whole system. Four parameters can be identified in system (4). One of them (namely, $\varepsilon$ ) depends on intrinsic diode parameters and thus will be kept constant during all the numerical experiment: $\varepsilon=5.4291 \times 10^{-4}$. Therefore, the bifurcation analysis of the system will be performed with respect to the control parameters $\rho, \alpha$, and $\mu$ (i.e., in terms of $R_{6}, R_{2}$, and $R_{3}$, resp.). Two of these parameters (namely, $\rho$ and $\alpha$ ) will be kept constant during all the numerical experiment: $\rho=1$ and $\alpha=1$. Only the parameter $\mu$ is tuneable. The values of electronic components used for both the numerical and experimental analyses are provided in the caption of Figure 1. It is obvious that system (4) can be written equivalently in the general jerk form as follows:

$$
\dddot{x}=J(\ddot{x}, \dot{x}, x)=-\mu \ddot{x}-\alpha \rho x-\alpha \varepsilon(\exp (\dot{x})-1) .
$$

This latter expression shows that our model belongs to the larger class of "elegant" jerk dynamical systems described 
in [25]. More interestingly, our model (4) represents one of the simplest autonomous $3 \mathrm{D}$ systems reported to date, capable of exhibiting asymmetric double strange attractors (see Sections 4 and 5) depending uniquely on the choice of initial conditions $[3,4,17]$.

\section{Analysis of the Model}

3.1. Dissipation and Existence of Attractors. The existence of attractors in our model could be examined by estimating the volume contraction rate [26-28] of the oscillator described in (4). We can recall that the volume of contraction rate of a continuous-time dynamical system is given by $\dot{x}=\varphi(X)$, where $X=(x, y, z)^{T}$ and $\varphi(X)=\left(\varphi_{1}(X), \varphi_{2}(X), \varphi_{3}(X)\right)^{T}$, and is given by

$$
\Upsilon=\nabla \cdot \varphi(X)=\frac{\partial \varphi_{1}}{\partial x}+\frac{\partial \varphi_{2}}{\partial y}+\frac{\partial \varphi_{3}}{\partial z} .
$$

We note that if $\Upsilon$ is a constant, then the time evolution in phase space is determined by $V(t)=V_{0} e^{\Upsilon t}$, where $V_{0}=$ $V(t=0)$. Thus, a negative value of $\Upsilon$ leads to a fast exponential shrink (i.e., damped) of the volume in state space, and the dynamical system is dissipative and can experience or develop attractors. For $\Upsilon=0$, phase-space volume is conserved and the dynamical system is conservative. When $\Upsilon$ is positive, the volume in phase space stretches out and therefore there exist only unstable fixed points or cycles or possibly repellors [26-28]; in other words, as time increased (i.e., for $t \rightarrow \infty$ ) the dynamics diverge if the initial conditions do not coincide exactly with one of the fixed points or stationary states. Considering our model, we have found that $\Upsilon=-\mu<0$ independently of the position $X=(x, y, z)^{T}$ in state space; hence, system (4) is dissipative and thus can support attractors.

3.2. Fixed Point Analysis. The equilibrium points [26-28] of (4) can be obtained by setting to zero the left-hand side. Obviously, $P_{0}(0,0,0)$ is the only fixed point of system (4). As mentioned above, system (4) possesses a single equilibrium point located at the origin of the system coordinates independently of the values of control parameters. The Jacobian matrix evaluated at the equilibrium point $P_{0}$ has the following expression:

$$
J=\left[\begin{array}{ccc}
0 & 1 & 0 \\
0 & 0 & \alpha \\
-\rho & -\varepsilon & -\mu
\end{array}\right] .
$$

Thus the stability of the trivial equilibrium point $P_{0}(0,0,0)$ is determined according to the real parts of the eigenvalues of the following characteristic equations:

$$
\begin{aligned}
\operatorname{det}(J-\lambda I) & =0, \\
\lambda^{3}+\mu \lambda^{2}+\varepsilon \alpha \lambda+\rho \alpha & =0,
\end{aligned}
$$

where $I$ represents the $3 \times 3$ identity matrix and $\lambda$ is the eigenvalue. A set of necessary and sufficient conditions for all the roots of (8a) and (8b) to have negative real parts is given by the well-known Routh-Hurwitz criterion expressed in the following form:

$$
\begin{array}{r}
\varepsilon, \alpha, \rho, \mu>0, \\
\varepsilon \mu-\rho>0 .
\end{array}
$$

$P_{0}$ was examined regarding the Routh-Hurwitz stability criterion and we have found that $P_{0}$ is stable only for $\mu>\mu_{c}=\rho / \varepsilon \approx 1841.909$. Meanwhile if the control parameter $\mu$ decreased beyond the critical value $\mu_{c}$, the state of nonoscillation can change. Therefore, Hopf bifurcation conditions have been deduced as follows:

$$
\begin{aligned}
\omega_{\text {Hopf }} & =\sqrt{\varepsilon \alpha} \\
\mu_{c} & =\frac{\rho}{\varepsilon} \\
\operatorname{Re} a l\left(\left.\frac{d \lambda}{d \mu}\right|_{\mu=\mu_{c}}\right) & =\frac{1}{2} \frac{-\varepsilon^{3} \alpha}{\left(\varepsilon^{3} \alpha+\rho^{2}\right)} \neq 0 .
\end{aligned}
$$

Equation (10a) defines the frequency of stable oscillations as well as the critical value of $\mu$ corresponding to the Hopf bifurcation of the system. From (10b), it can be noted that the transversality condition is always met since $\mu$ is positive control parameter. From the above discussion, it follows that, in the regime of regular or chaotic oscillations, the single fixed point $P_{0}$ is unstable, and thus the system generates self-excited oscillations $[29,30]$. For instance, considering the particular case of $\mu=1.100$ for which the system displays a strange attractor (see Section 4) the eigenvalues evaluated at $P_{0}$ are $\lambda_{1}=-1.5280$ and $\lambda_{2,3}=0.2140 \pm j 0.7802$. This clearly shows that the fixed point $P_{0}$ is unstable in the chaotic regime, which is typical of self-excited oscillations.

\section{Numerical Study}

4.1. Route to Chaos. To define different scenarios/routes to chaos in our model and to investigate the rich variety of bifurcation modes that can be observed in the jerk system, system (4) has been numerically solved using standard fourthorder Runge-Kutta integration scheme technique. Dynamical study of the system begins by analyzing possible states of fixed points, their stability, and bifurcations which happen under the control of corresponding parameters of system components. For this paper, the time step is chosen such that $\Delta t \leq 0.005$ for each set of parameters and the computations are carried out using variables and constant parameters in extended mode. For each parameters setting, the system is integrated for a sufficiently long time and the transient is discarded. Two indicators are exploited to characterize the type of transition leading to chaos. The first indicator is the bifurcation diagram, the second indicator being the graph of Lyapunov exponents' spectra. Regarding the last tool, the dynamics of the system are categorized using its Lyapunov exponents which are computed numerically with the help of the algorithm described in [31]. The investigation of the sensitivity of the system, with respect to a single 


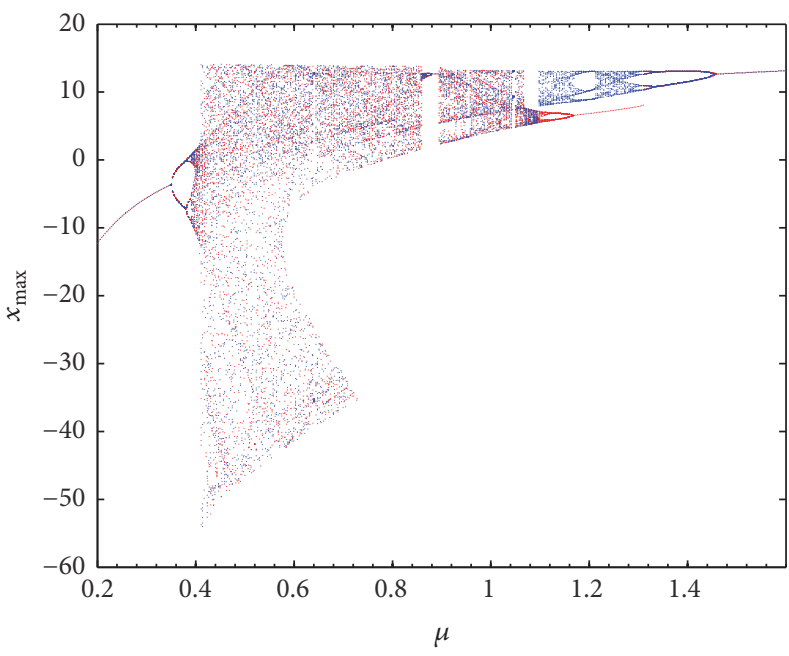

(a)

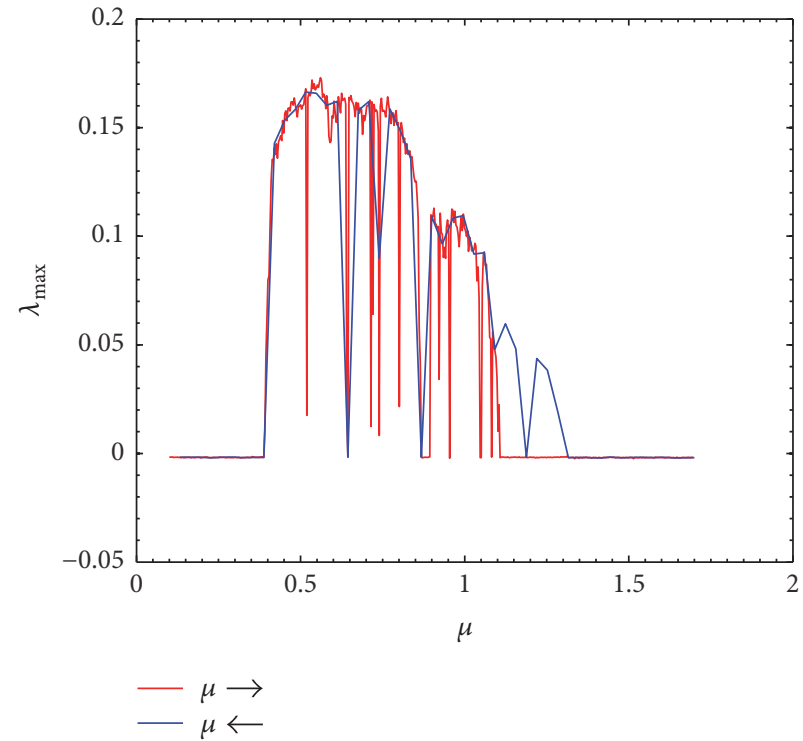

(b)

FIGURE 2: Bifurcation diagrams (a) showing local maxima of the coordinate $x(\tau)$ versus $\mu$ of the jerk attractor in two directions (red dot indicates first direction from down to up and the blue dot indicates the second direction from up to down) and the corresponding graphs (b) of $1 \mathrm{D}$ largest numerical Lyapunov $\left(\lambda_{\max }\right)$ exponent plotted in the range $0.1 \leq \mu \leq 1.7$. A positive exponent $\left(\lambda_{\max }>0\right)$ indicates chaos, while regular states are characterized with negative values of Lyapunov exponent $\left(\lambda_{\max }<0\right)$.

control parameter $\mu$, has been carried out by varying $\mu$ within the interval range from 0.1 to 1.7 . As the control parameter is varying, we have discovered that very abundant and remarkable bifurcation scenarios were exhibited by the jerk system under our consideration. In Figures 2(a) and 2(b), respectively, sample findings exhibiting bifurcation diagram for different value of $\mu$ and the corresponding spectrum of Lyapunov exponents are presented. The bifurcation diagram is obtained by graphing local maxima of coordinate $x$ versus control parameter (two directions: increasing and decreasing) inside narrow steps interval $0.1 \leq \mu \leq 1.7$. The ultimate state for every iteration of the control parameter is used as initial state for subsequent iteration. Two categories of data corresponding, respectively, to increasing (red) and decreasing (blue) values of $\mu$ are superimposed in Figure 2. This method of gathering curves represents a simple way to localize the window in which the system develops multiple coexisting attractors' behavior (see Section 4.2). Chaos motion is achieved progressively within the chaotic oscillator with respect to the control parameter $\mu$. Perioddoubling bifurcations, reverse period-doubling sequence, and periodic windows can easily be identified in the graphs of Figures 2(a) and 2(b). Using the same parameters settings in Figure 2, various numerical computations of phase portraits (Figure 3) of the oscillator and corresponding time series (Figure 3) were obtained confirming different transitions depicted previously.

The Hopf type bifurcation and the period-doubling scenario to chaos are observed when using $\alpha$ and $\rho$, respectively, as bifurcation control parameters for $\mu=0.5$ (see Figure 4). To observe the phenomenon of period-doubling scenario to chaos, we fix $\mu=0.5$ and plot the bifurcation diagram.
4.2. Coexistence of Attractors. As mentioned above, the system behavior is different depending on whether the control parameter is swept in upward and downward direction, expressing the hysteretic behavior of the model. To provide a better illustration, we show in Figure 5 two zooms of the bifurcation of Figure 2.

One can remark that for $1.1 \leq \mu \leq 1.31$ the system displays two different attractors depending solely on choice of the initials conditions. This is perfectly illustrated in Figure 6 where some sample pair of coexisting solutions are presented: (a) coexistence of a pair of asymmetric strange attractors, (b) coexistence of a period-2 limit cycle with a chaotic attractor, (c) coexistence of period-3 cycle with a period-1 cycle, (d) coexistence of a chaotic attractor with a period-1 cycle, and (e) a period- 4 cycle with a period-1 cycle (see Table 1 ).

To gain more insight about the complexity of the attractor depicted in Figure 6(a), various three-dimensional projections are presented in Figure 7(a). In the same line as Figure $7(\mathrm{~b})$, time traces of the coordinate $x(\tau)$ to illustrate the crisis induced intermittency experienced by the system. Furthermore, the Poincaré section of the attractor computed is shown in Figures 7(c) and 7(d). The shape of this Poincaré section is characteristic of chaotic attractors in Figures $6(\mathrm{a}(\mathrm{i}))$ and $6(\mathrm{a}(\mathrm{ii}))$. Briefly recall that the Poincaré plot contains points at the intersections between the phase-space trajectory and Poincaré (plane). An appropriate location of the plane in the state space is chosen in order to obtain details of structure of the attractor [28].

The basin of attraction is the set of initial conditions whose trajectories converge to the given attractor, and it is usually exploited to provide more information about the coexisting attractors. The structure of the basin boundaries 

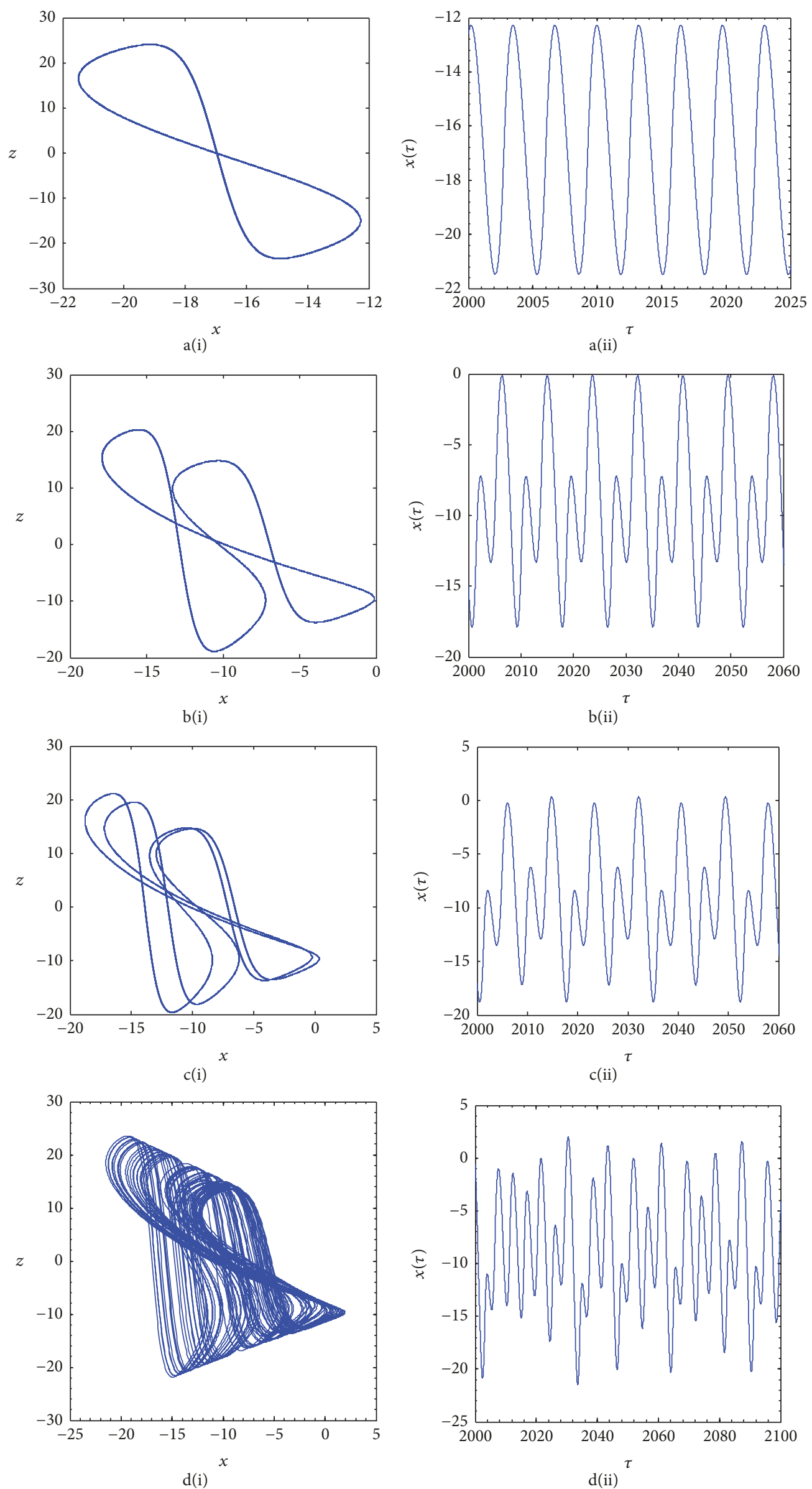

Figure 3: Continued. 


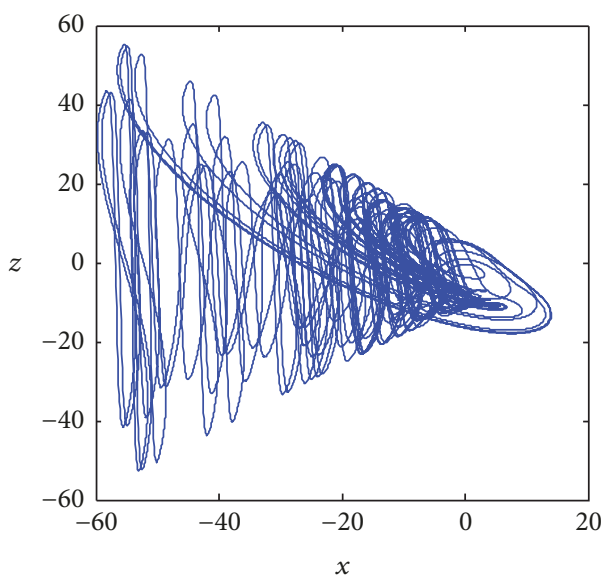

$\mathrm{e}(\mathrm{i})$

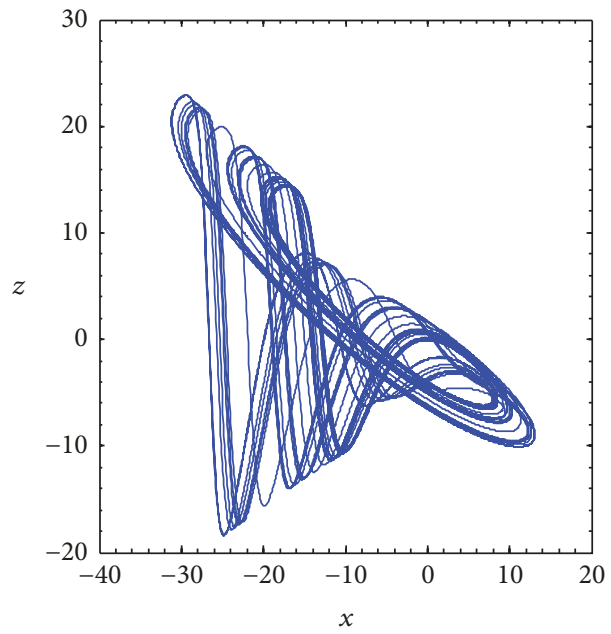

$\mathrm{f}(\mathrm{i})$

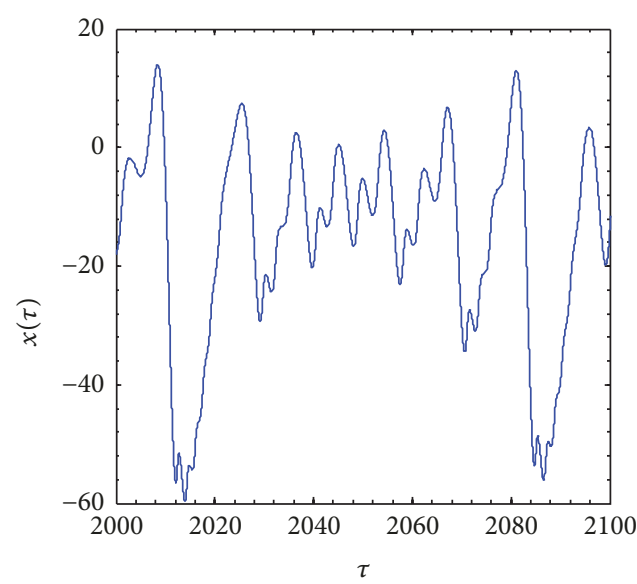

e(ii)

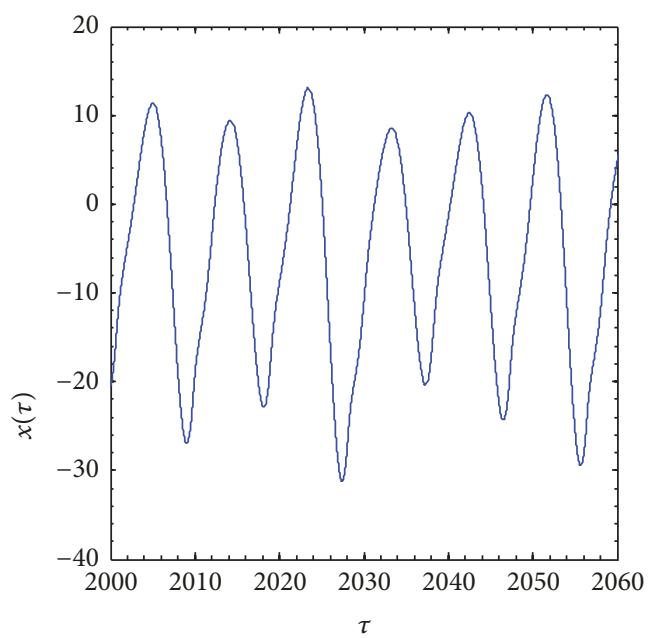

f(ii)

Figure 3: Numerical phase portraits in $X Z$ plane (left) of the system showing routes to chaos (in terms of the control parameter $\mu$ ) and corresponding time traces (right) of the coordinate $x(\tau)$ for varying $\mu$ : (a) period-1 cycle for $\mu=0.165$; (b) period-2 cycle for $\mu=0.381$; (c) period- 4 cycle for $\mu=0.385$; (d) spiralling-like (Feigenbaum) chaotic attractor for $\mu=0.406$; (e) screw-like (Shilnikov) chaotic attractor for $\mu=0.420$; (f) another screw-like (Shilnikov) chaotic attractor for $\mu=1.100$.

TABLE 1: Sample forms of coexisting attractors in terms of control parameter $\mu$.

\begin{tabular}{lcc}
\hline Value of control parameter & $\begin{array}{c}\text { Initial conditions } \\
(x(0), y(0), z(0))\end{array}$ & Figure 6 \\
\hline$\mu=1.100$ & $(1,0,0)$ & a(i): screw-like (Shilnikov) chaotic attractor \\
& $(1.167,0,0)$ & a(ii): spiralling- like (Feigenbaum) chaotic attractor \\
$\mu=1.130$ & $(5,0,0)$ & b(i): screw-like (Shilnikov) chaotic attractor \\
& $(1.417,0,0)$ & b(ii): period-2 cycle \\
$\mu=1.200$ & $(5,0,0)$ & $c(i)$ period-3 cycle \\
& $(-1,-1,-1)$ & $c($ ii): period-1 cycle \\
$\mu=1.250$ & $(5,0,0)$ & d(i): screw-like (Shilnikov) chaotic attractor \\
& $(-3.917,0,0)$ & d(ii): period-1 cycle \\
& $(5,0,0)$ & e(i): period-4 cycles \\
\end{tabular}



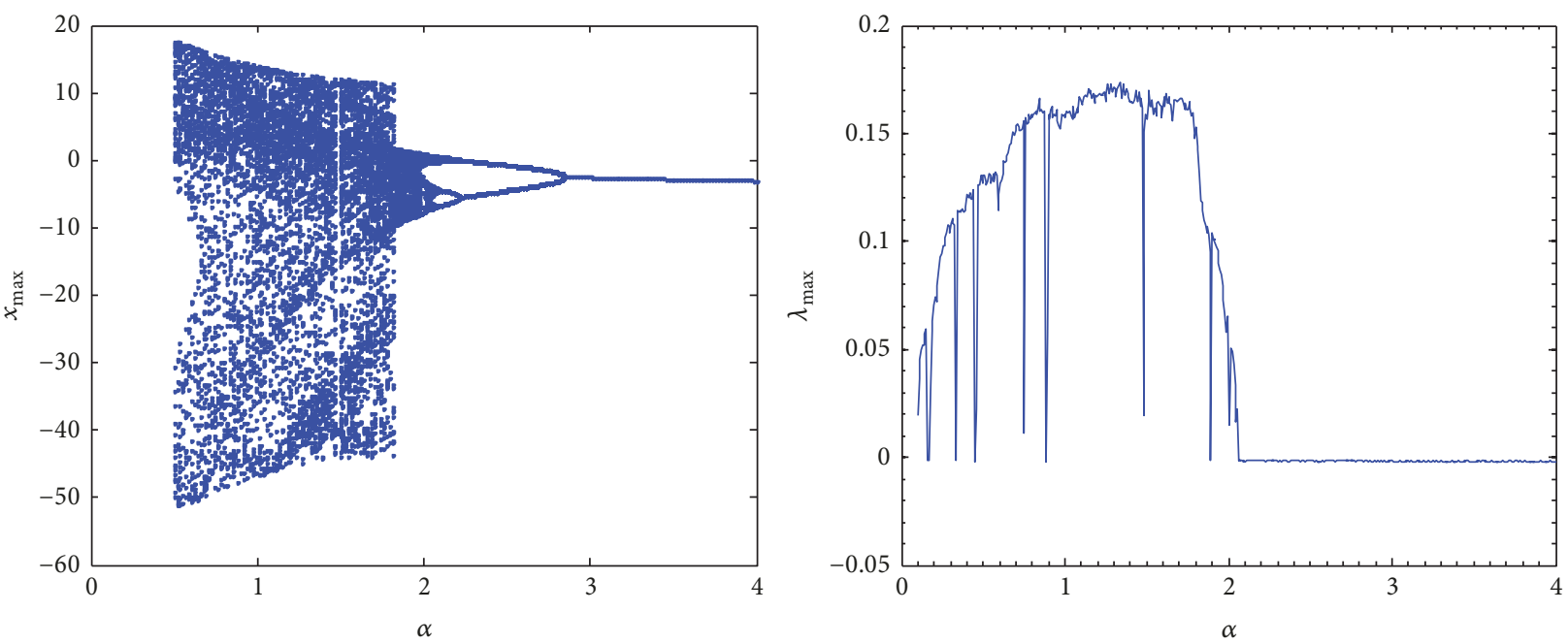

(a)
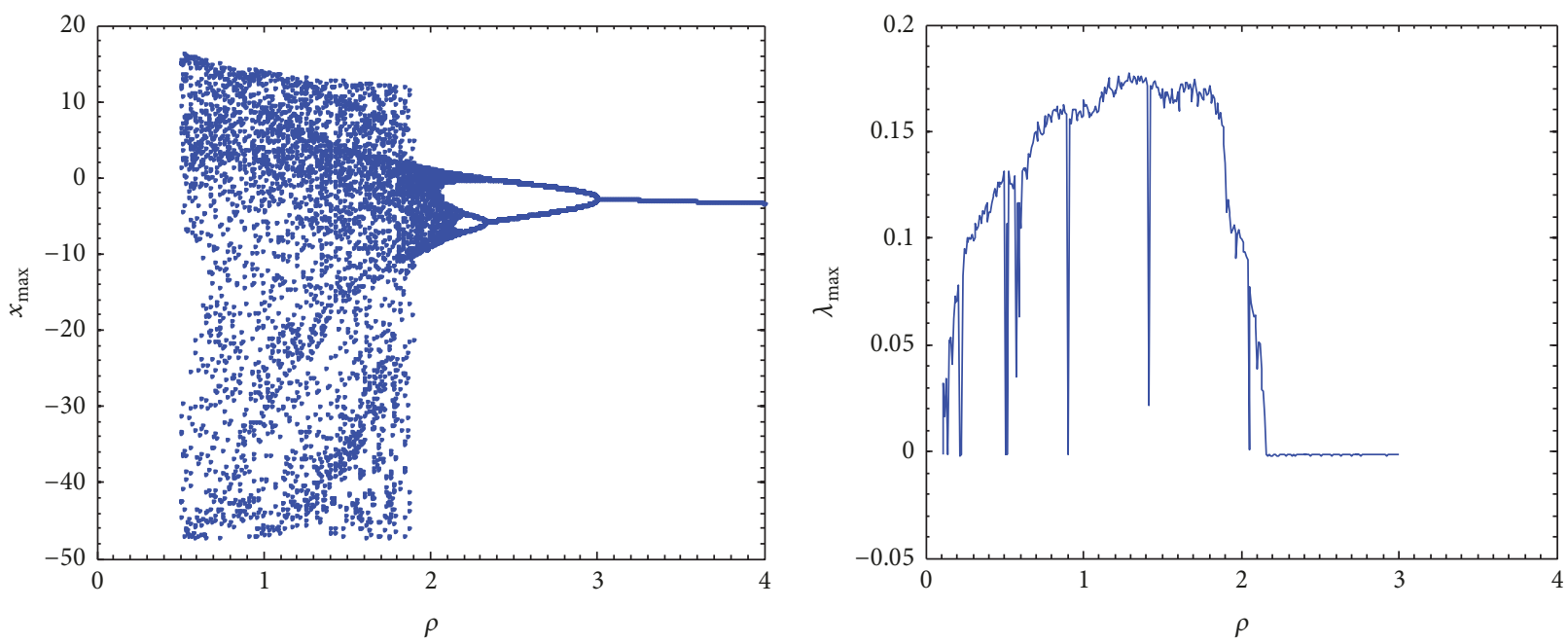

(b)

Figure 4: Bifurcation diagrams. (a): local maxima of the coordinate $x(\tau)$ versus $\alpha$ of the jerk attractor for $\mu=0.5$ and the corresponding graphs of 1D largest numerical Lyapunov $\left(\lambda_{\max }\right)$ exponent plotted in the range $0.5 \leq \alpha \leq 4$; (b): local maxima of the coordinate $x(\tau)$ versus $\rho$ of the jerk attractor for $\mu=0.5$ and the corresponding graphs (b) of 1D largest numerical Lyapunov $\left(\lambda_{\max }\right)$ exponent plotted in the range $0.5 \leq \rho \leq 3.5$.

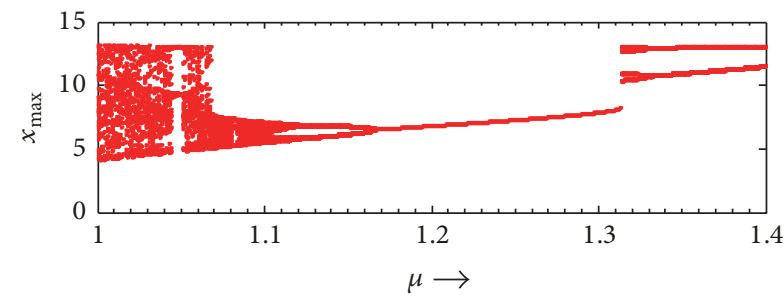

(a)

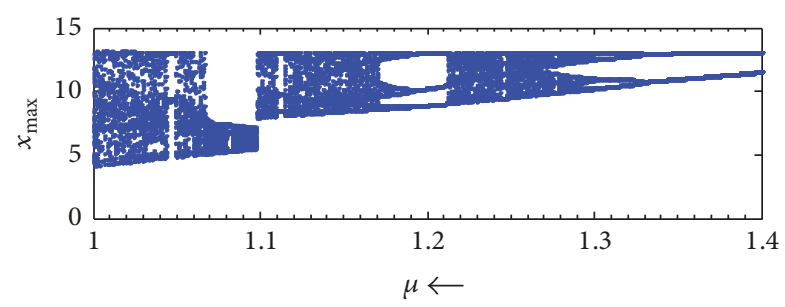

(b)

FIGURE 5: Enlargement of the bifurcation diagram of Figure 2 showing the region in which the system exhibits multiple coexisting attractors. This region corresponds to values of $\mu$ in the range: $1 \leq \mu \leq 1.4$. Two sets of data corresponding, respectively, to (a) increasing (upward sweep in red) and (b) decreasing (downward sweep in blue) values of the bifurcation control parameter are superimposed. 


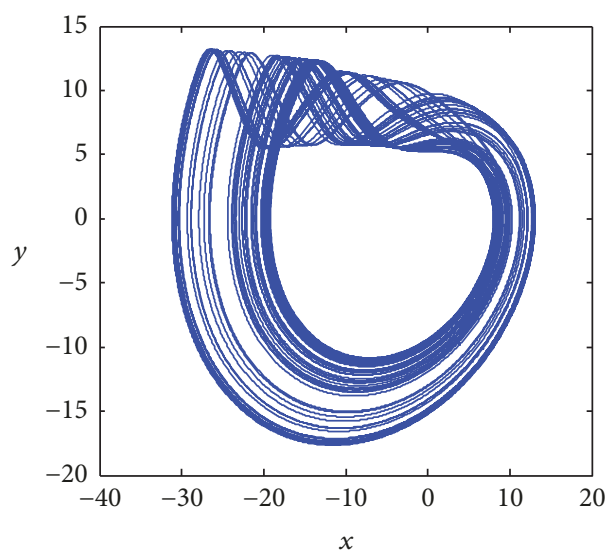

$\mathrm{a}(\mathrm{i})$

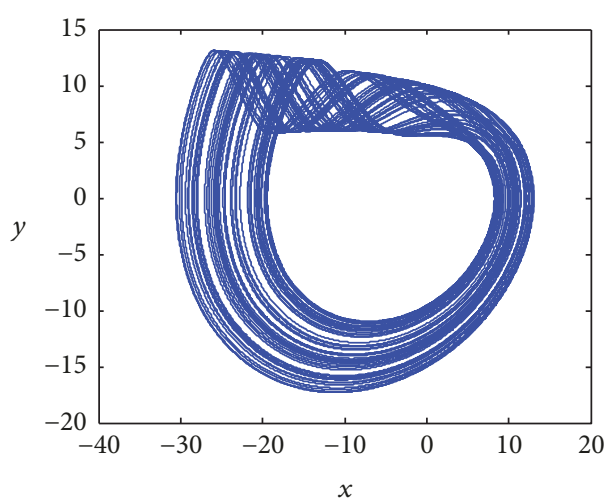

$\mathrm{b}(\mathrm{i})$

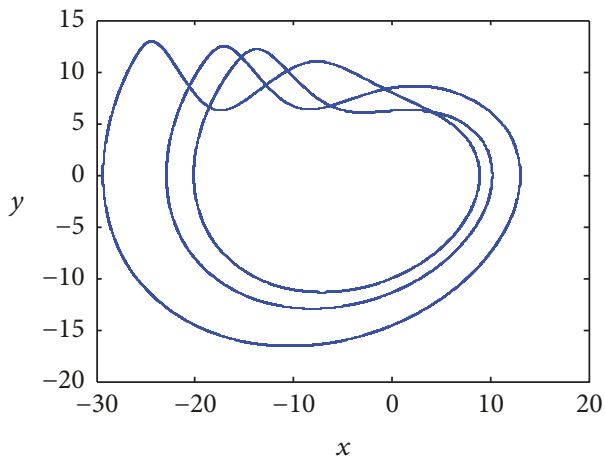

c(i)

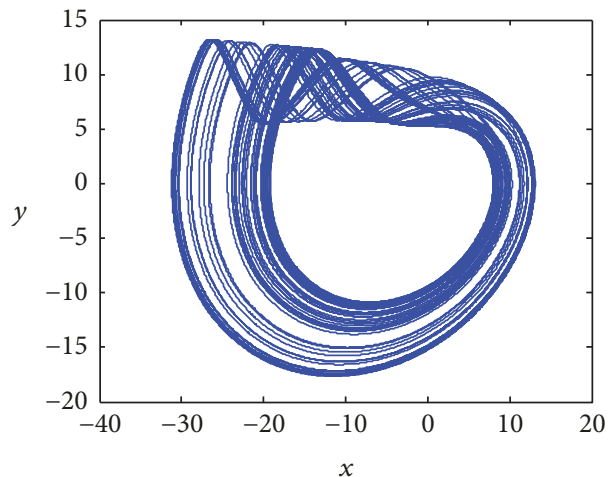

$\mathrm{d}(\mathrm{i})$

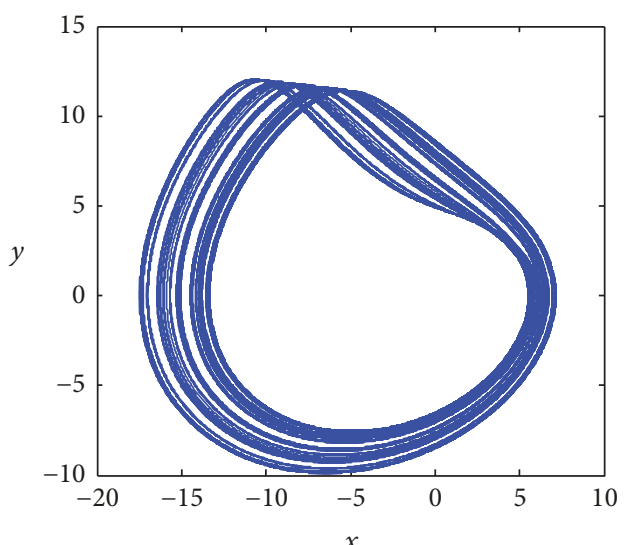

a(ii)

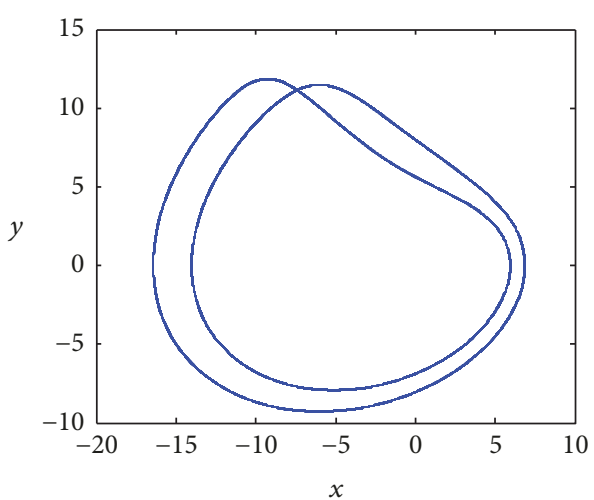

$\mathrm{b}(\mathrm{ii})$

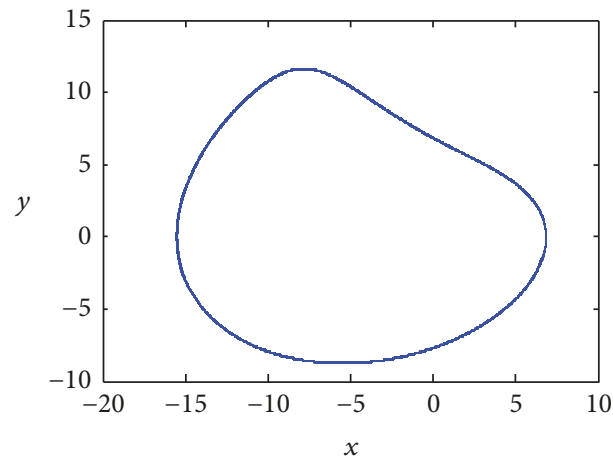

c(ii)

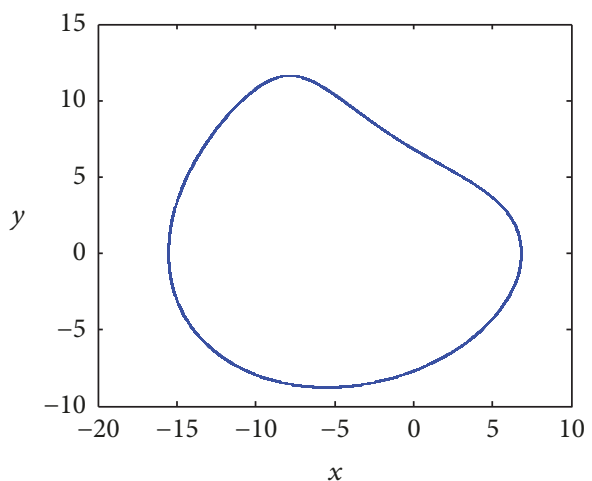

d(ii)

Figure 6: Continued. 


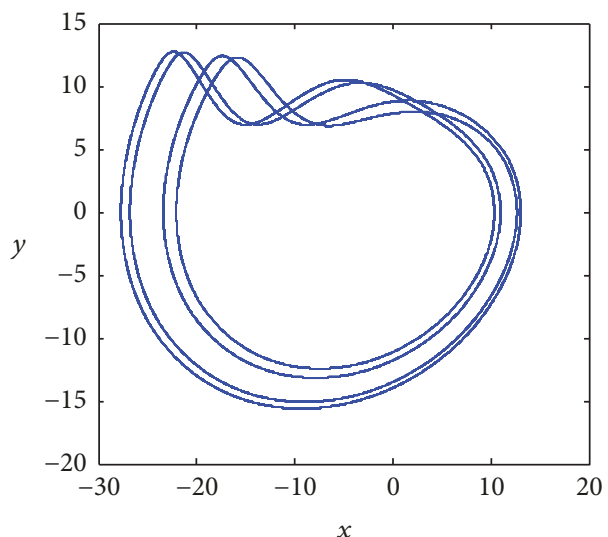

e(i)

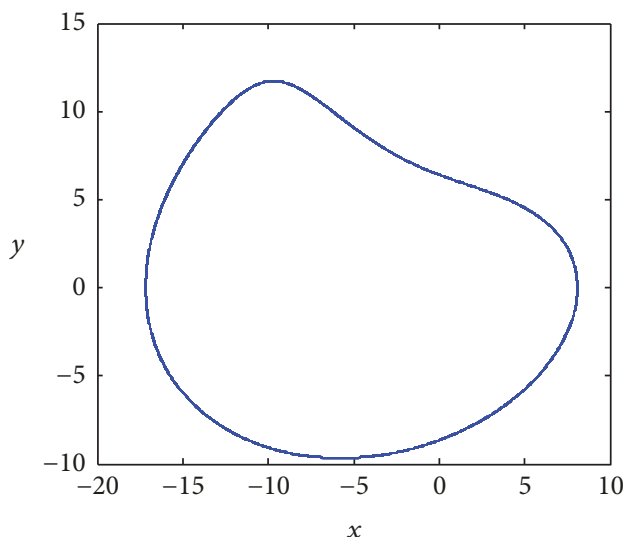

e(ii)

FIGURE 6: Numerical phase portraits showing the coexistence of multiple (two) solutions for different values of the control parameter $\mu$. The values of the control parameters as well as initial conditions are listed in Table 1.

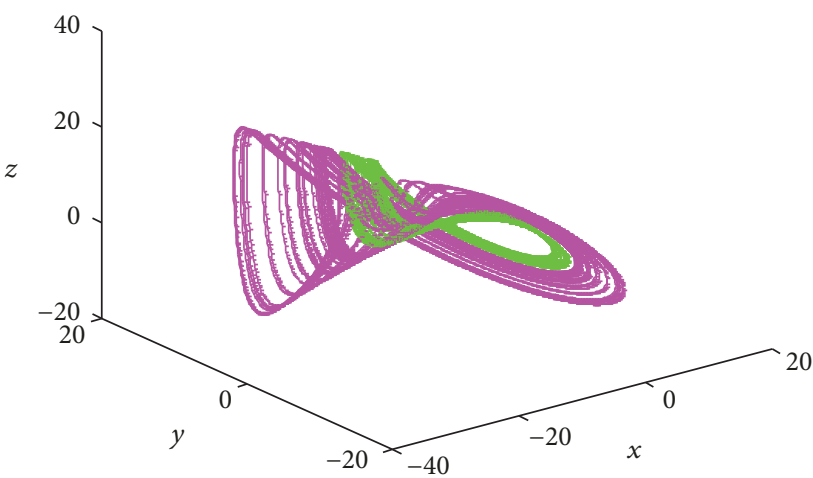

(a)

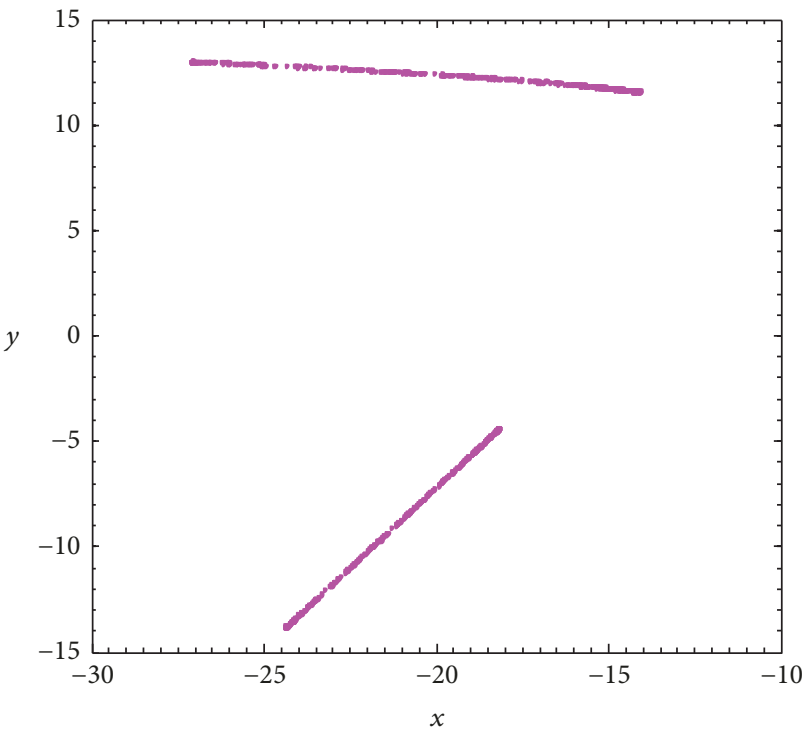

(c)

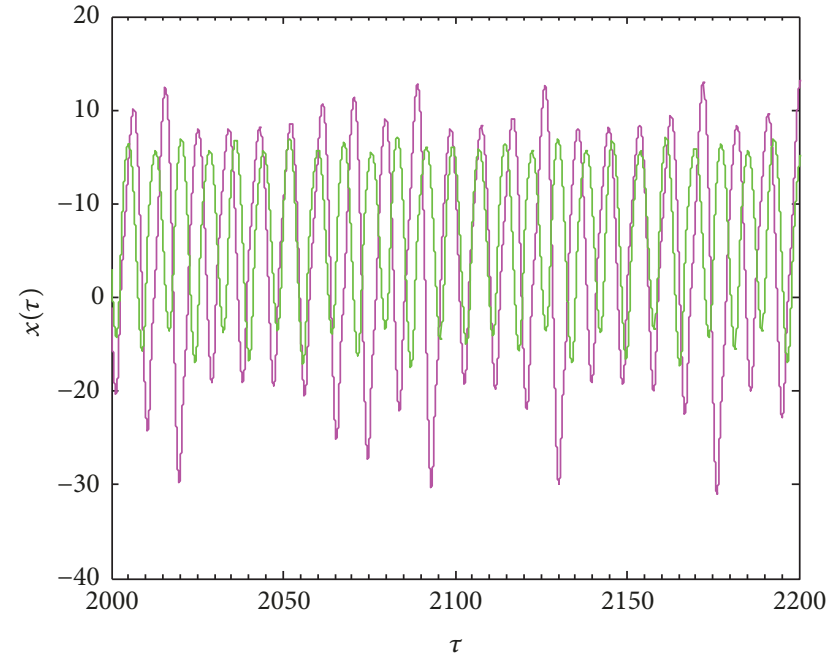

(b)

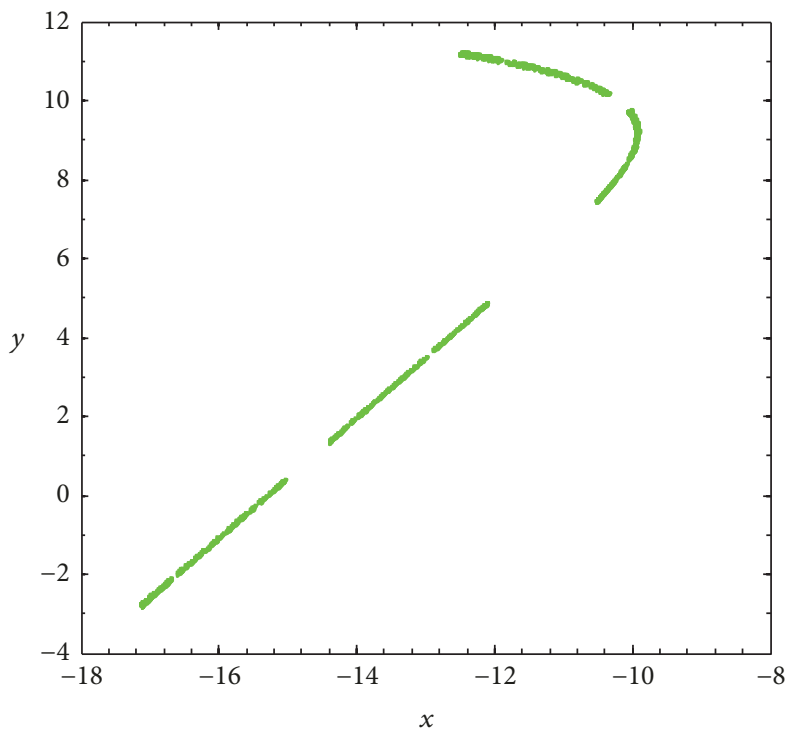

(d)

FIGURE 7: Three-dimensional view of the attractor projected onto the $(x, y, z)$ space of the system showing the coexistence of single-band chaotic attractor (attractor (screw-like Shilnikov) a(i) in magenta with a different single-band chaotic attractor (spiralling-like Feigenbaum) a(ii) in green color. (b) is the corresponding time traces of the coordinate $x(\tau)$ ) for a(i) in magenta and a(ii) in green. (c) is the corresponding single-sided Poincaré section in the plane $z=0$ for a(i). (d) is the corresponding single-sided Poincaré section in the plane $z=0$ for a(ii). 


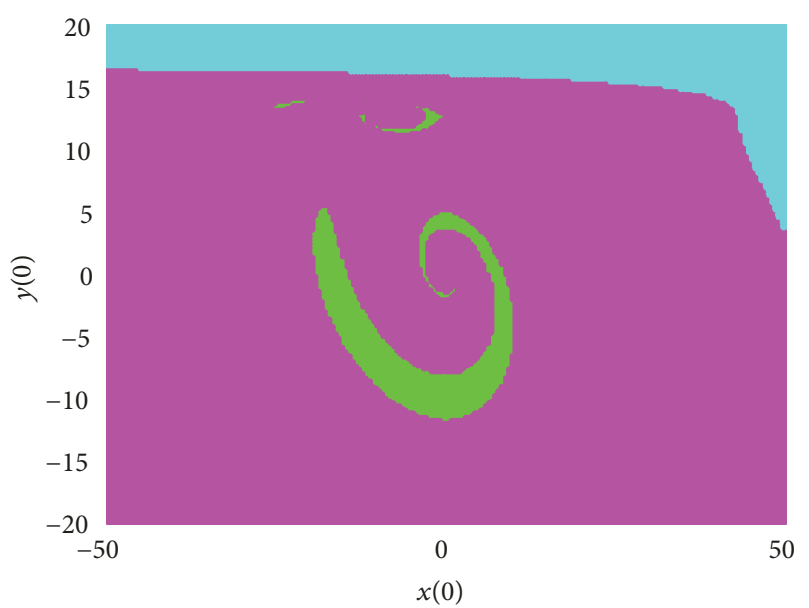

(a)

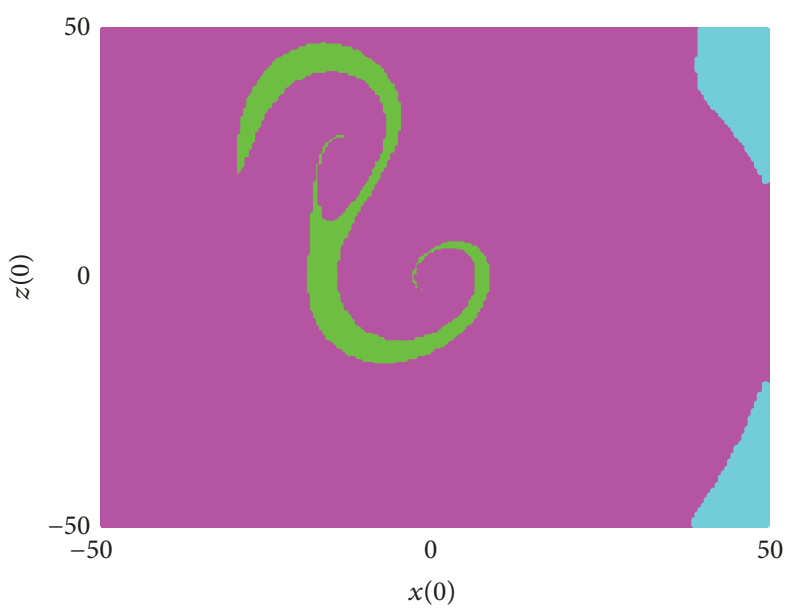

(b)

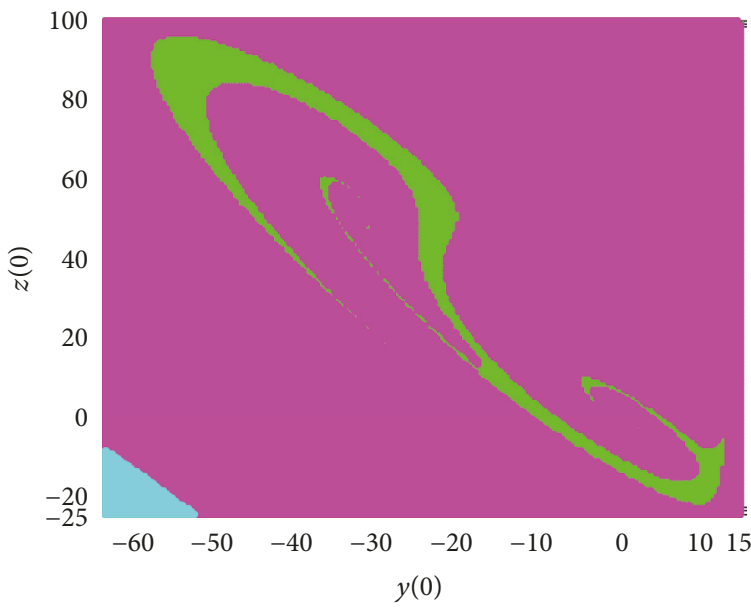

(c)

Figure 8: Structure of the three cross sections of the basin of attraction with $z(0)=0$ and $(x(0), y(0))$ in the ranges $-60 \leq x(0) \leq 50$ and $-25 \leq y(0) \leq 100$ obtained for $\mu=1.100$. We present the basin of attraction for the chaotic coexistence (a): magenta regions correspond to asymmetric single-band chaotic attractor (screw-like Shilnikov), while green ones are related to another single-band chaotic attractor (spiralling-like Feigenbaum). Blue zones correspond to unbounded solutions. These regions represent initial conditions that lead to each chaotic trajectory.

is clearly illustrated in Figure 8 where three cross sections of the basins of attraction are depicted, respectively, for $x(0)=$ $0, y(0)=0$, and $z(0)=0$, associated with the spiralling or Feigenbaum strange attractors (green) and the screw or Shilnikov strange attractors (magenta). Blue regions correspond to unbounded dynamics. It should be mentioned that, according to the best of the authors' knowledge, the striking phenomenon of coexisting asymmetric strange attractors also found in some special cases of nonlinear systems such as the Colpitts oscillator [3], Newton-Leipnik systems [17], and the hyperchaotic oscillator with gyrators [4] has not yet been reported in any other jerk system and thus represents an enriching contribution related to the dynamic behavior of this large class of nonlinear systems. Provided that the occurrence of multiple attractors represents an additional source of randomness [32], some obvious potential applications include, for instance, chaos based secure communication as well as random signal generation. However, this singular type of behavior is not desirable in general and justifies the need for control. Detailed analysis on this line is beyond the scope of this contribution. Therefore, interested readers are guided to the interesting review paper on control of multistability presented in [33].

\section{Experimental Study}

Following the above theoretical analysis, it is predicted that the jerk system under investigation can display very rich and complex behaviors. Our goal in this section is to validate the theoretical results obtained previously by performing an experimental study of the practical system [18]. To this end, the schematic diagram of Figure 1 is constructed on a breadboard. The circuit is built using TL084 op. amplifiers type with a symmetric $\pm 15 \mathrm{~V}$ DC voltage supply. The same values of electronics components used for the numerical study are kept here to enable the comparison process. The 


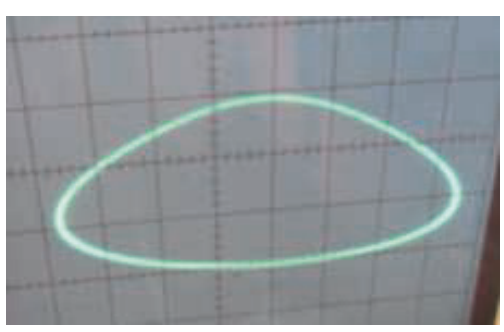

a(i)

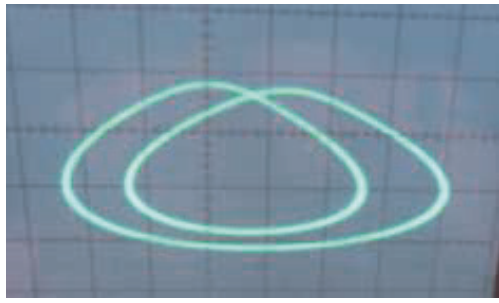

b(i)

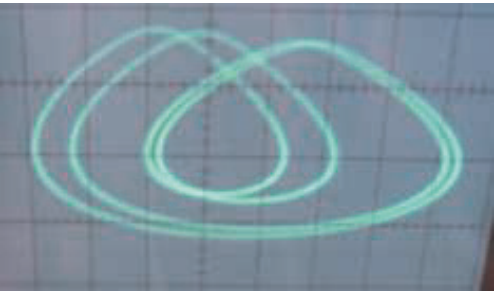

c(i)

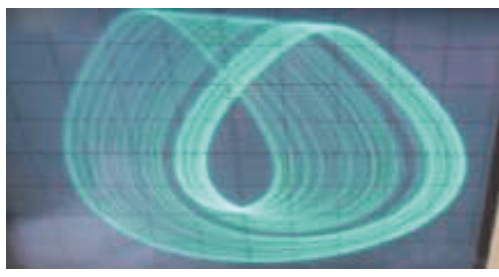

d(i)

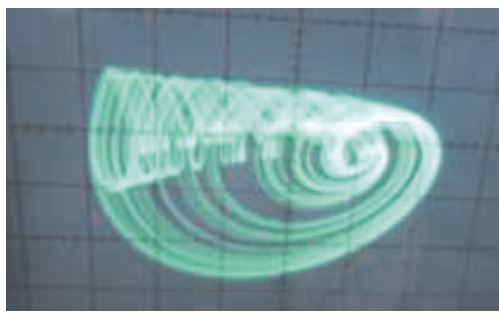

e(i)

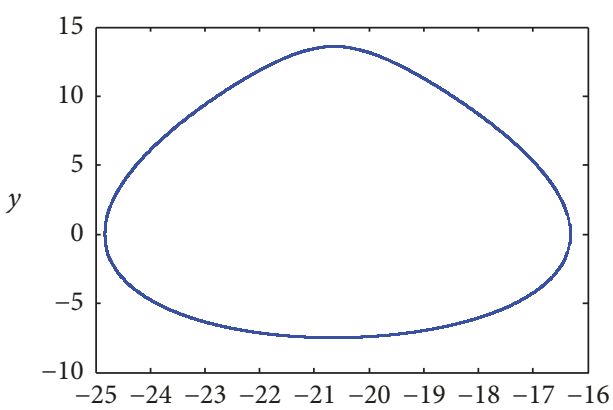

a(ii)

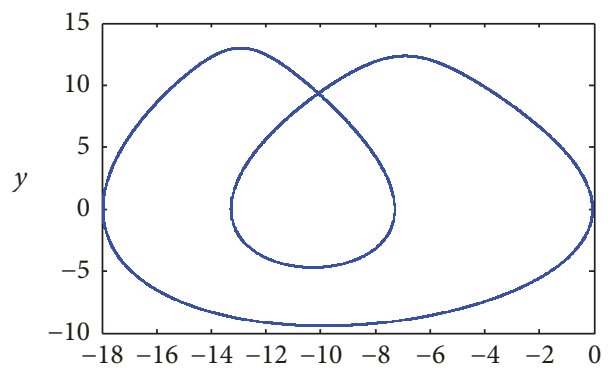

b(ii)

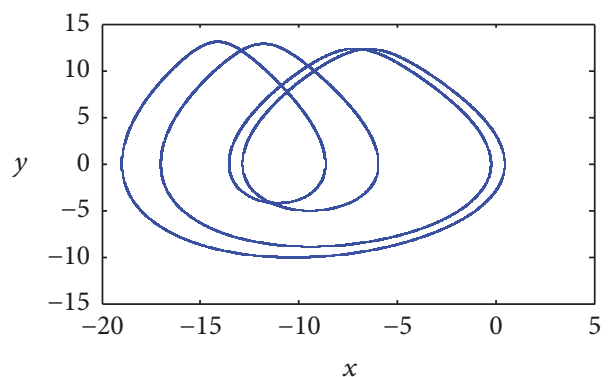

c(ii)

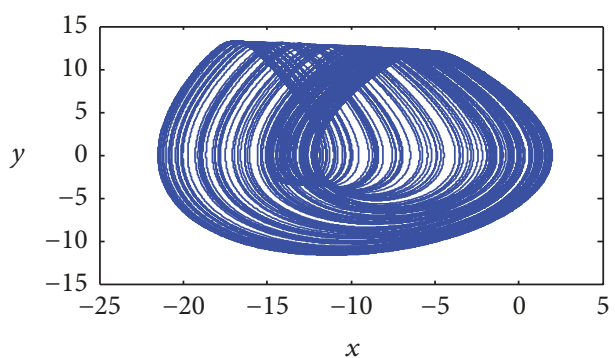

$\mathrm{d}(\mathrm{ii})$

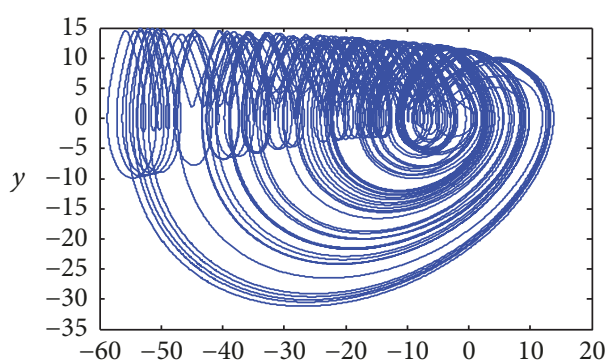

e(ii) ${ }^{x}$

FIGURE 9: Continued. 


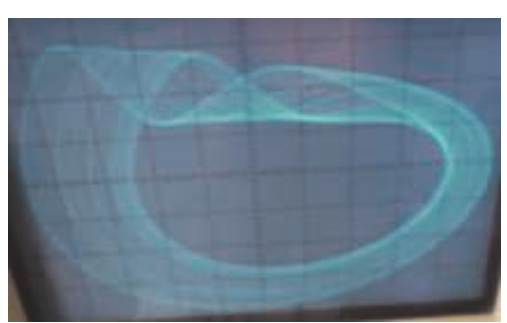

$\mathrm{f}(\mathrm{i})$

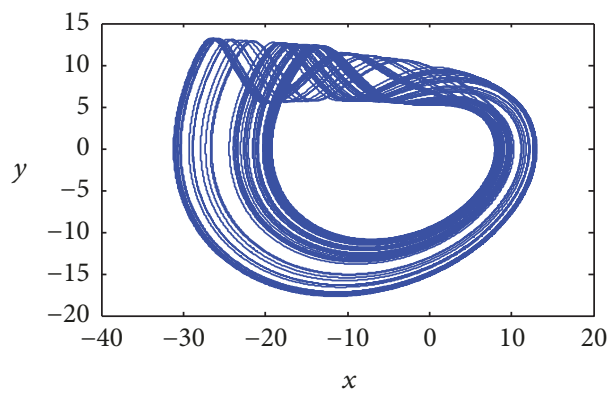

$\mathrm{f}(\mathrm{ii})$

Figure 9: Experimental phase portraits (left) obtained from the circuit using a dual-trace oscilloscope in $X Y$; the corresponding numerical phase portraits (right) showing the period-doubling routes to chaos in the system for varying $R_{3}$ (i.e., $\mu$ ): (a) period-1 cycle for $R_{3}=$ $60.3 \mathrm{k} \Omega$ (i.e., $\mu=0.165$ ); (b) period-2 cycle for $R_{3}=26.2 \mathrm{k} \Omega$ (i.e., $\mu=0.381$ ); (c) period- 4 cycle for $R_{3}=25.97 \mathrm{k} \Omega$ (i.e., $\mu=0.385$ ); (d) spiralling-like (Feigenbaum) chaotic attractor for $R_{3}=24.60 \mathrm{k} \Omega$ (i.e., $\mu=0.406$ ); (e) screw-like (Shilnikov) chaotic attractor for $R_{3}=23.81 \mathrm{k} \Omega$ (i.e., $\mu=0.420$ ); (f) another screw-like (Shilnikov) chaotic attractor for $R_{3}=9.09 \mathrm{k} \Omega$ (i.e., $\mu=1.100$ ).

experimental results are obtained by slowly increasing or decreasing the control resistor $R_{3}$ (i.e., parameter $\mu$ ) and plotting phase-space trajectories $(Y, X)$ using a dual-trace oscilloscope in the $X Y$ mode. When slowly monitoring the control resistor $R_{3}$, we found that the experimental jerk circuit experiences various types of bifurcation as predicted by theoretical results. For $R_{3}=60,3 \mathrm{k} \Omega$, a period-1 limit cycle is observed. When $R_{3}$ is gradually decreased, the complete sequence of bifurcation reported above is observed, that is, sequence of period-doubling bifurcation to chaos followed by the reverse period-doubling leading to a regular period1 motion (see Figure 9). This is clearly illustrated by sample experimental phase portraits in Figure 9 showing the real behavior of the jerk circuit under consideration. In light of the pictures in Figure 9, it can be seen that the real circuit demonstrates the same bifurcation sequences as observed during the numerical study.

Those two-dimensional projections of asymmetric singleband chaotic attractor in Figure 9 highlight the complexity of the system. However, this attractor is simple chaotic. A comparison between experimental (Figure 10) and numerical results (Figure 6) related to the presence of five coexisting attractors shows a perfect agreement. More interestingly, the hysteretic behavior is observed in the experimental jerk circuit by increasing the value of $R_{3}$ in such a way that the circuit moves from the state of period-1 motion to another period-1 motion via the sequence of period-doubling and reverse bifurcation and then decreasing the value of the same resistor to move back to the original state of period-1 motion. Thus the system follows a different path while $R_{3}$ is decreased rather than $R_{3}$ being increased, reminiscent of a hysteresis. Once more a very good qualitative agreement can be captured between numerical and experimental results. However, a slight discrepancy that may be attributed to the precision on the values of electronic components as well as the simplifying assumptions considered during the modeling process (i.e., ideal diode model, ideal op. amplifier) can be noted between numerical and experimental results (see caption of Figure 10). Similarly, we have also verified the dynamical behaviors, the transitions from period-doubling bifurcation to chaos, the reverse bifurcation sequence, and the coexistence of multiple attractors evaluated through PSpice simulation when varying the value of the control resistor $R_{3}$. However, we have avoided the inclusion of the simulation results for the sake of brevity.

Some windows of periodic behaviors sandwiched within chaotic domains are also noted in experiment. To experimentally provide evidence of coexisting multiple attractors in the jerk circuit, the control resistor is fixed as follows: $R_{3}=$ $9.09 \mathrm{k} \Omega$ (i.e., $\mu=1.100$ ); $R_{3}=8.85 \mathrm{k} \Omega$ (i.e., $\mu=1.130$ ); $R_{3}=8.33 \mathrm{k} \Omega$ (i.e., $\mu=1.200$ ); $R_{3}=8.00 \mathrm{k} \Omega$ (i.e., $\mu=$ $1.250) ; R_{3}=7.63 \mathrm{k} \Omega$ (i.e., $\mu=1.310$ ). When switching on and off the power supply (and thereby randomly selecting initial states), one can obtain five pairs of coexisting asymmetric solutions shown in Figure 10.

\section{Concluding Remarks}

A relatively simple chaotic jerk circuit, recently introduced by [18], has been analyzed in this work. Using bifurcation diagrams as arguments, the dynamics of the systems have been categorized in terms of their parameters. Period-doubling bifurcations, periodic windows, and coexisting bifurcations were found when monitoring the system parameters. More interestingly a distinguishing feature of this jerk circuit not previously observed in the pioneering work of Sprott [18] is the possibility of double asymmetric strange attractors (i.e., Shilnikov chaos and Feigenbaum chaos) for a wide range of parameters, depending solely on the choice of initial states. Also, we would like to stress that the jerk circuit/system studied in this work according to the best of our knowledge represents the simplest prototype among the few cases of lower dimensional system capable of exhibiting such type of behavior (e.g., Rossler system, Colpitts oscillator, Newton-Leipnik system, and hyperchaotic oscillator with gyrators) and thus deserves dissemination [34]. Laboratory experimental measurements were in very good agreement with the theoretical analyses. The presence of coexisting strange attractors represents another source of randomness, with potential utility in random signal generation, chaos communication, and radar systems. It should be pointed 


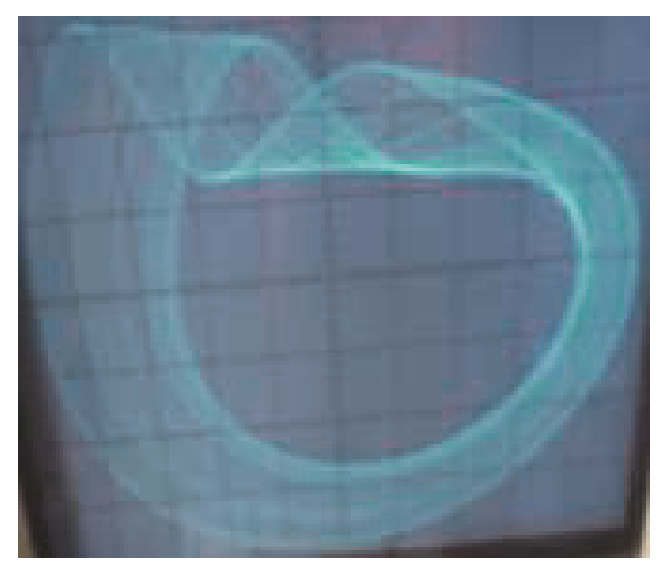

a(i)

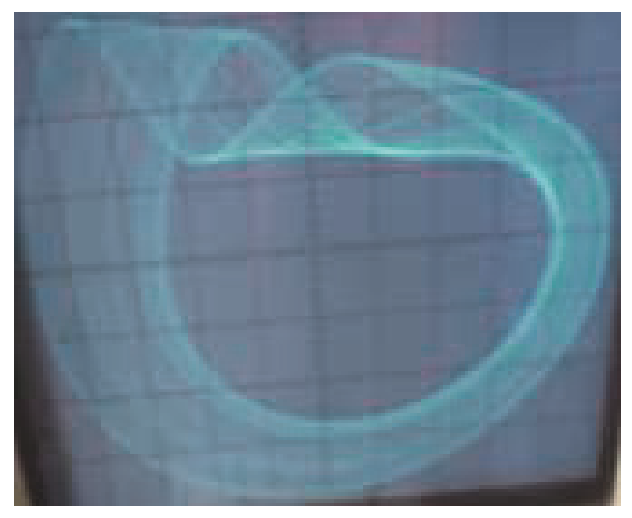

$b(i)$

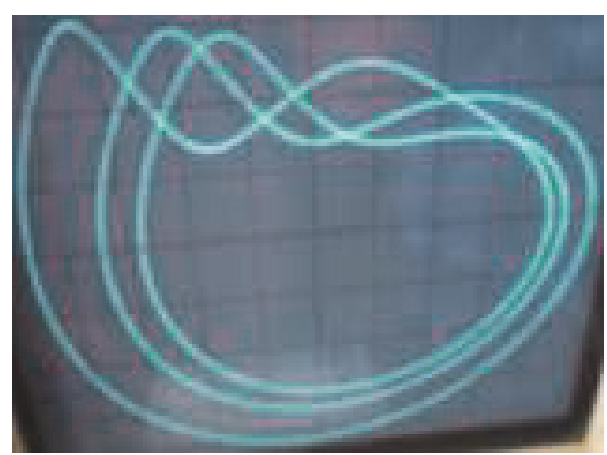

c(i)

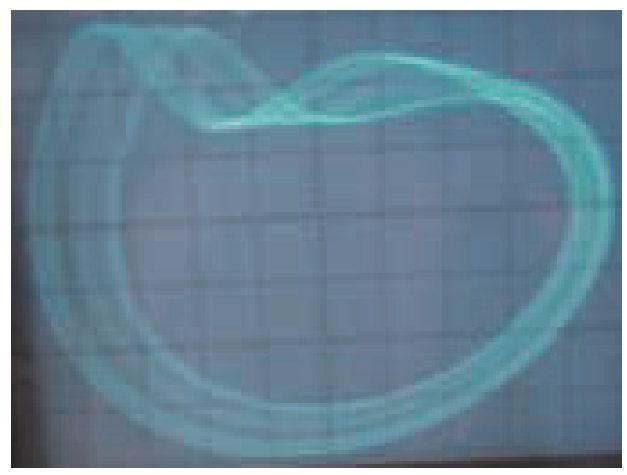

d(i)

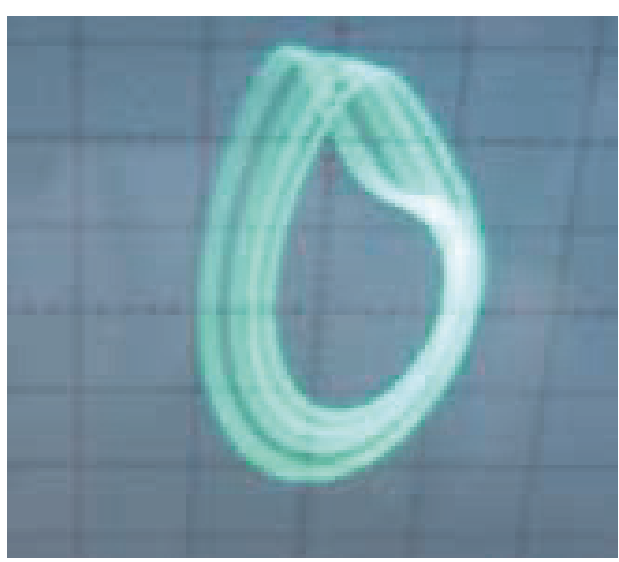

a(ii)

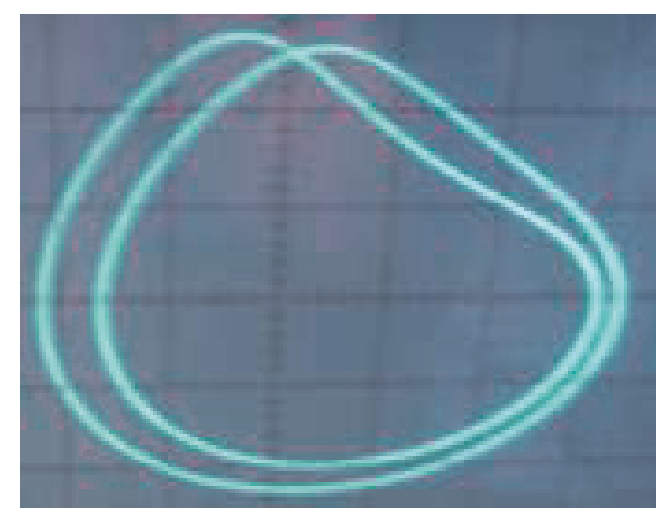

b(ii)

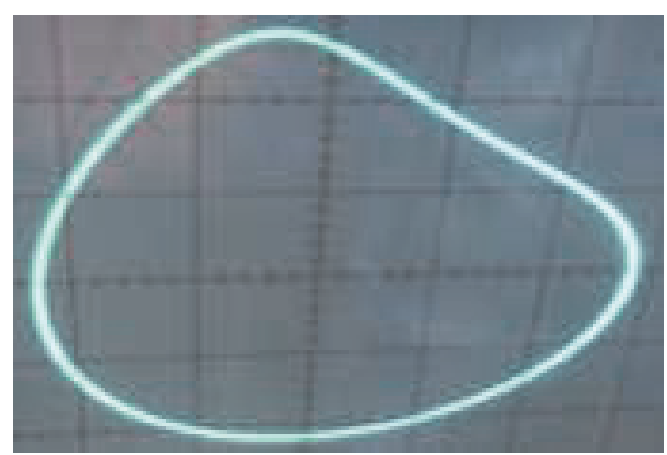

c(ii)

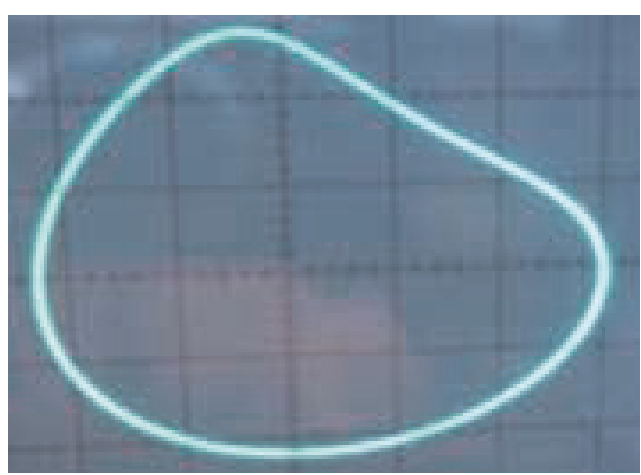

d(ii)

Figure 10: Continued. 


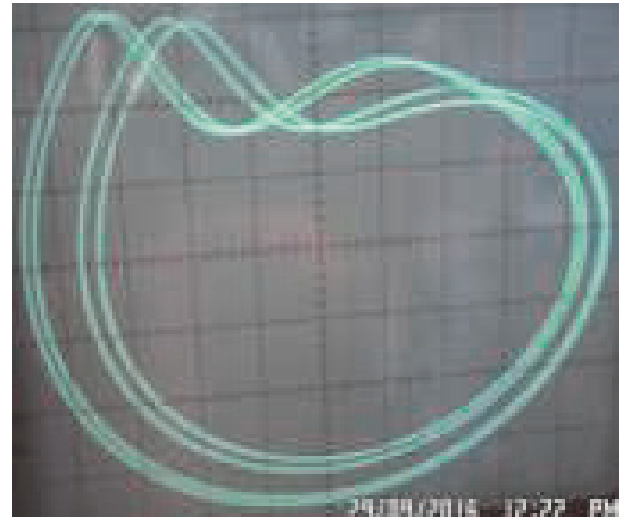

e(i)

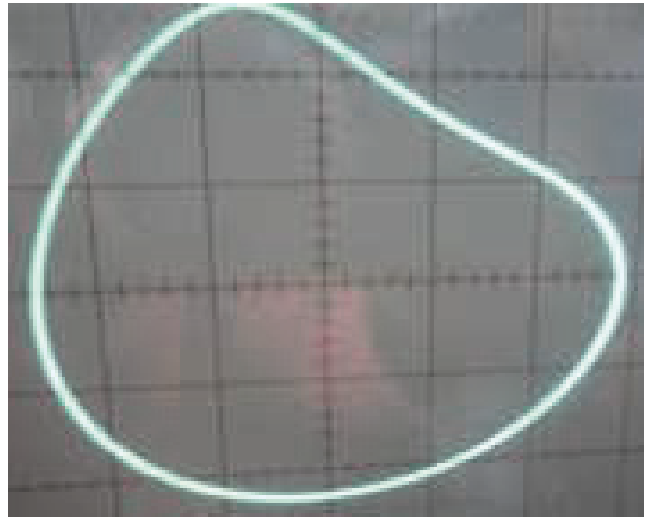

e(ii)

FIGURE 10: Experimental phase portraits showing the coexistence of multiple (two) solutions for different values of the control parameter $R_{3}$ (i.e., $\mu$ ). We have five pairs of mismatch coexisting asymmetric solutions: (a) screw-like (Shilnikov) chaotic attractor a(i) coexisting with a spiralling- like (Feigenbaum) chaotic attractor a(ii) for $R_{3}=9.09 \mathrm{k}$ (i.e., $\mu=1.100$ ); (b) screw-like (Shilnikov) chaotic attractor b(i) coexisting with a period- 2 cycle $\mathrm{b}$ (ii) for $R_{3}=8.85 \mathrm{k}$ (i.e., $\mu=1.130$ ); (c) period-3 cycle $\mathrm{c}(\mathrm{i})$ coexisting with a period-1 cycle $\mathrm{c}(\mathrm{ii})$ for $R_{3}=8.33 \mathrm{k}$ (i.e., $\mu=1.200$ ); (d) screw-like (Shilnikov) chaotic attractor $\mathrm{d}$ (i) coexisting with a period-1 cycle $\mathrm{d}$ (ii) for $R_{3}=8.00 \mathrm{k}$ (i.e., $\mu=1.250$ ); (e) period- 4 cycles e(i) coexisting with a period-1 cycle e(ii) for $R_{3}=7.63 \mathrm{k}$ (i.e., $\mu=1.310$ ). Each pair of solutions appears randomly in experiment when switching on and off the power supply. The scales are $X=0.2 \mathrm{~V} / \mathrm{div}$ and $Y=0.2 \mathrm{~V} / \mathrm{div}$ for all pictures. Note the perfect similarity of those pictures with the numerical phase portraits of Figure 6.

out that an infinite number of equilibria could be obtained in the jerk circuit under consideration via replacing the semiconductor diode (the nonlinear component) with a flux controlled memristor $[14,35]$. Such types of systems are more suited to develop the phenomenon of extreme multistability $[11,36,37]$ involving the coexistence of an infinite number of attractors for the same parameter setting, depending only on the choice of initial conditions. Research along this line is under consideration and the results will be the material of an upcoming publication.

\section{Conflicts of Interest}

The authors declare that there are no conflicts of interest regarding the publication of this paper.

\section{References}

[1] B. Bao, T. Jiang, Q. Xu, M. Chen, H. Wu, and Y. Hu, "Coexisting infinitely many attractors in active band-pass filter-based memristive circuit," Nonlinear Dynamics, vol. 86, no. 3, pp. 1711-1723, 2016.

[2] J. Kengne, Z. T. Njitacke, A. Nguomkam Negou, M. Fouodji Tsostop, and H. B. Fotsin, "Coexistence of Multiple Attractors and Crisis Route to Chaos in a Novel Chaotic Jerk Circuit," International Journal of Bifurcation and Chaos, vol. 26, no. 5, Article ID 1650081, 19 pages, 2016.

[3] G. M. Maggio, O. De Feo, and M. P. Kennedy, "Nonlinear analysis of the Colpitts oscillator and application to design," IEEE Transactions on Circuits and Systems I: Fundamental Theory and Applications, vol. 46, pp. 1118-1130, 1999.

[4] J. Kengne, "Coexistence of chaos with hyperchaos, period-3 doubling bifurcation, and transient chaos in the hyperchaotic oscillator with gyrators," International Journal of Bifurcation and
Chaos in Applied Sciences and Engineering, vol. 25, no. 4, Article ID 1550052, 17 pages, 2015.

[5] V. Vaithianathan and J. Veijun, "Coexistence of four different attractors in a fundamental power system model," IEEE Transactions on Circuits and Systems I, vol. 46, pp. 405-409, 1999.

[6] S. Banerjee, "Coexisting attractors, chaotic saddles, and fractal basins in a power electronic circuit," IEEE Transactions on Circuits and Systems I: Fundamental Theory and Applications, vol. 44, no. 9, pp. 847-849, 1997.

[7] V. N. Chizhevsky, "Coexisting attractors in a $\mathrm{CO}_{2}$ laser with modulated losses," Journal of Optics B: Quantum and Semiclassical Optics, vol. 2, no. 6, pp. 711-711, 2000.

[8] J. M. Cushing, S. M. Henson, and C. C. Blackburn, "Multiple mixed attractors in a competition model," Journal of biological dynamics, vol. 1, pp. 347-362, 2007.

[9] A. Massoudi, M. G. Mahjani, and M. Jafarian, "Multiple attractors in Koper-Gaspard model of electrochemical," Journal of Electroanalytical Chemistry, vol. 647, no. 1, pp. 74-86, 2010.

[10] S. Morfu, B. Nofiele, and P. Marquié, "On the use of multistability for image processing," Physics Letters, Section A: General, Atomic and Solid State Physics, vol. 367, no. 3, pp. 192-198, 2007.

[11] H. Sun, S. K. Scott, and K. Showalter, "Uncertain destination dynamics," Physical Review E, vol. 60, no. 4, pp. 3876-3880, 1999.

[12] M. S. Patel, U. Patel, A. Sen et al., "Experimental observation of extreme multistability in an electronic system of two coupled Rössler oscillators," Physical Review E - Statistical, Nonlinear, and Soft Matter Physics, vol. 89, no. 2, Article ID 022918, 2014.

[13] C. Hens, S. K. Dana, and U. Feudel, "Extreme multistability: attractor manipulation and robustness," Chaos. An Interdisciplinary Journal of Nonlinear Science, vol. 25, 053112.1-7, no. 5, 2015.

[14] B. Bocheng, X. Quan, B. Han, and C. Mo, "Extreme multistability in a memristive circuit," Electronics Letters, vol. 52, no. 12, pp. 1008-1010, 2016.

[15] J. Kengne, Z. Njitacke Tabekoueng, V. Kamdoum Tamba, and A. Nguomkam Negou, "Periodicity, chaos, and multiple 
attractors in a memristor-based Shinriki's circuit," Chaos. An Interdisciplinary Journal of Nonlinear Science, vol. 25, no. 10, Article ID 103126, 2015.

[16] J. C. Sprott, "Simplest chaotic flows with involutional symmetries," International Journal of Bifurcation and Chaos in Applied Sciences and Engineering, vol. 24, no. 1, Article ID 1450009, pp. 1-9, 2014.

[17] R. B. Leipnik and T. A. Newton, "Double strange attractors in rigid body motion with linear feedback control," Physics Letters A, vol. 86, no. 2, pp. 63-87, 1981.

[18] J. C. Sprott, "A new chaotic jerk circuit," IEEE Transactions on Circuits and Systems II: Express Briefs, vol. 58, no. 4, pp. 240243, 2011.

[19] J. C. Sprott, "Some simple jerk functions," American Journal of Physics, vol. 65, no. 6, pp. 537-543, 1997.

[20] J. C. Sprott, "Simple chaotic systems and circuits," American Journal of Physics, vol. 68, no. 8, pp. 758-763, 2000.

[21] R. Eichhorn, S. J. Linz, and P. Hänggi, "Simple polynomial classes of chaotic jerky dynamics," Chaos, Solitons \& Fractals, vol. 13, no. 1, pp. 1-15, 2002.

[22] J. Kengne, Z. T. Njitacke, and H. B. Fotsin, "Dynamical analysis of a simple autonomous jerk system with multiple attractors," Nonlinear Dynamics. An International Journal of Nonlinear Dynamics and Chaos in Engineering Systems, vol. 83, no. 1-2, pp. 751-765, 2016.

[23] M. P. Hanias, G. Giannaris, A. Spyridakis, and A. Rigas, "Time series analysis in chaotic diode resonator circuit," Chaos, Solitons and Fractals, vol. 27, no. 2, pp. 569-573, 2006.

[24] D. W. Sukov, M. E. Bleich, D. J. Gauthier, and J. E. Socolar, "Controlling chaos in a fast diode resonator using extended time-delay auto-synchronization: Experimental observations and theoretical analysis," Chaos, vol. 7, no. 4, pp. 560-576, 1997.

[25] B. Munmuangsaen, B. Srisuchinwong, and J. C. Sprott, "Generalization of the simplest autonomous chaotic system," Physics Letters A, vol. 375, no. 12, pp. 1445-1450, 2011.

[26] S. H. Strogatz, Nonlinear Dynamics and Chaos, vol. 78, Reading, Addison-Wesley, 1994.

[27] J. Argyris, G. Faust, and M. Haase, An exploration of chaos, North-Holland Publishing Co., Amsterdam, 1994.

[28] A. H. Nayfeh and B. Balachandran, Applied Nonlinear Dynamics: Analytical, Computational and Experimental Methods, Wiley Series in Nonlinear Science, John Wiley \& Sons, New York, USA, 1995.

[29] G. A. Leonov and N. V. Kuznetsov, "Hidden attractors in dynamical systems: from hidden oscillations in HilbertKolmogorov, Aizerman, and KALman problems to hidden chaotic attractor in Chua circuits," International Journal of Bifurcation and Chaos in Applied Sciences and Engineering, vol. 23, no. 1, Article ID 1330002, 69 pages, 2013.

[30] G. A. Leonov, N. V. Kuznetsov, and T. N. Mokaev, "Homoclinic orbits, and self-excited and hidden attractors in a Lorenzlike system describing convective fluid motion," The European Physical Journal Special Topics, vol. 224, no. 8, pp. 1421-1458, 2015.

[31] A. Wolf, J. B. Swift, H. L. Swinney, and J. A. Vastano, "Determining Lyapunov exponents from a time series," Physica D: Nonlinear Phenomena, vol. 16, no. 3, pp. 285-317, 1985.

[32] X. Luo, M. Small, M.-F. Danca, and G. Chen, "On a dynamical system with multiple chaotic attractors," International Journal of Bifurcation and Chaos in Applied Sciences and Engineering, vol. 17, no. 9, pp. 3235-3251, 2007.
[33] A. N. Pisarchik and U. Feudel, "Control of multistability," Physics Reports. A Review Section of Physics Letters, vol. 540, no. 4, pp. 167-218, 2014.

[34] J. C. Sprott, "A proposed standard for the publication of new chaotic systems," International Journal of Bifurcation and Chaos, vol. 21, no. 9, pp. 2391-2394, 2011.

[35] B. C. Bao, H. Bao, N. Wang, M. Chen, and Q. Xu, "Hidden extreme multistability in memristive hyperchaotic system," Chaos, Solitons and Fractals, vol. 94, pp. 102-111, 2017.

[36] C. N. Ngonghala, U. Feudel, and K. Showalter, "Extreme multistability in a chemical model system," Physical Review E-Statistical, Nonlinear, and Soft Matter Physics, vol. 83, no. 5, Article ID 056206, 2011.

[37] C. Hens, R. Banerjee, U. Feudel, and S. K. Dana, "How to obtain extreme multistability in coupled dynamical systems," Physical Review E, vol. 85, Article ID 035202, 2012. 


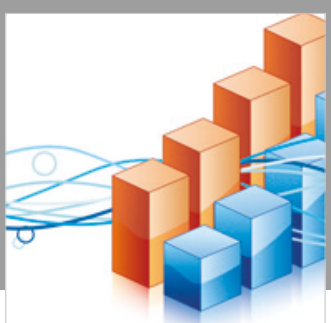

Advances in

Operations Research

\section{-n-m}
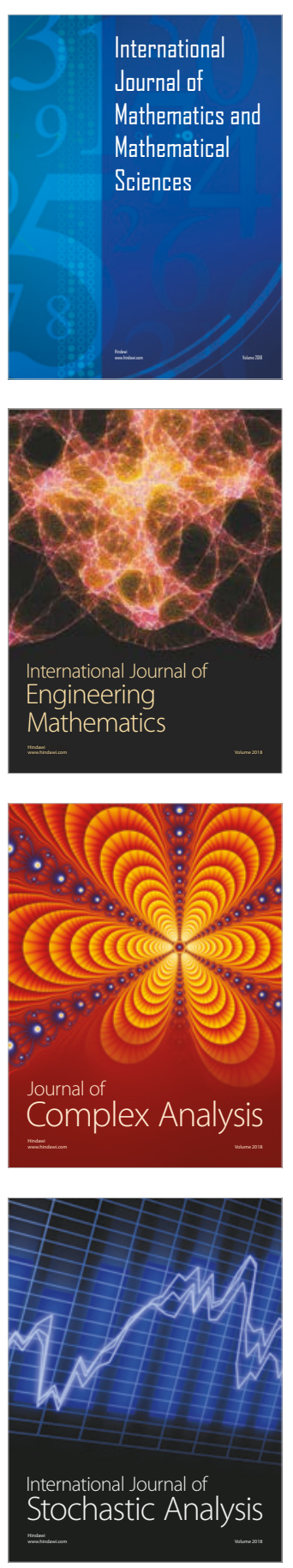
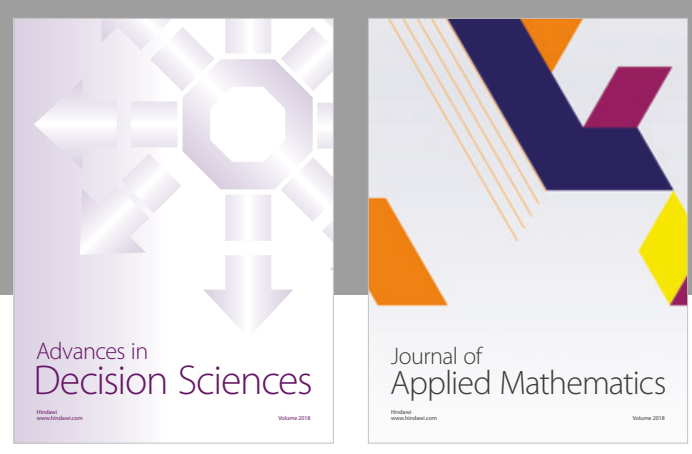

Journal of

Applied Mathematics
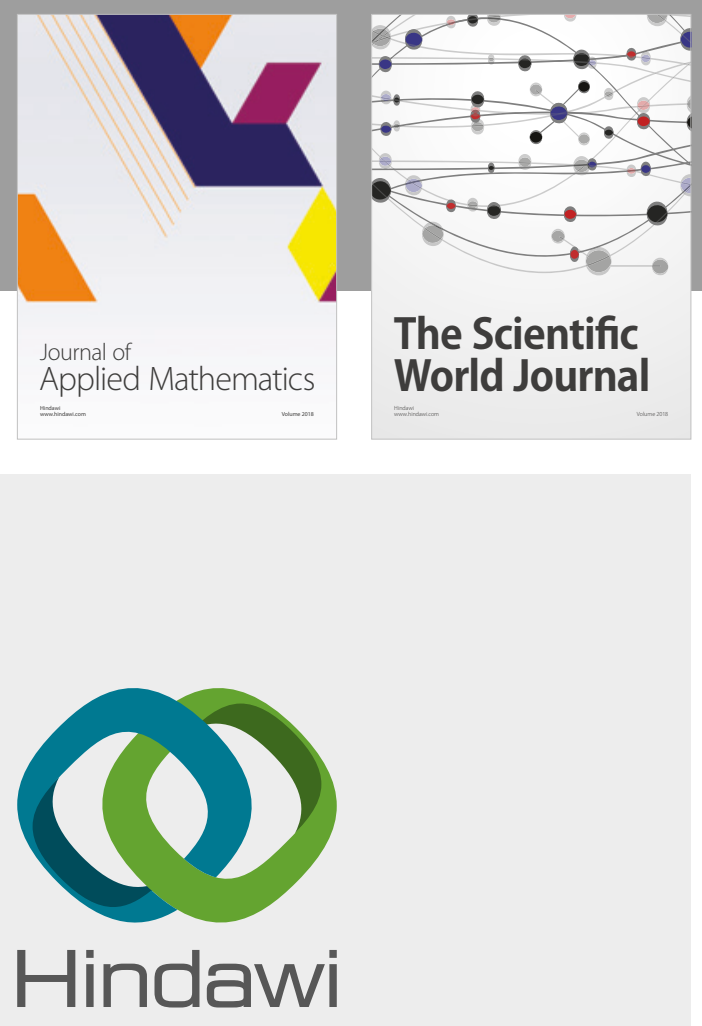

Submit your manuscripts at

www.hindawi.com

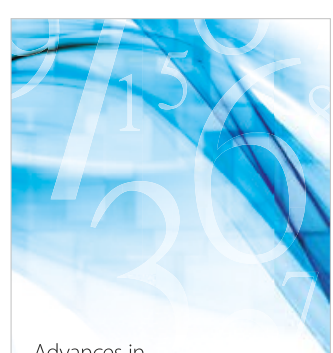

Advances in
Numerical Analysis
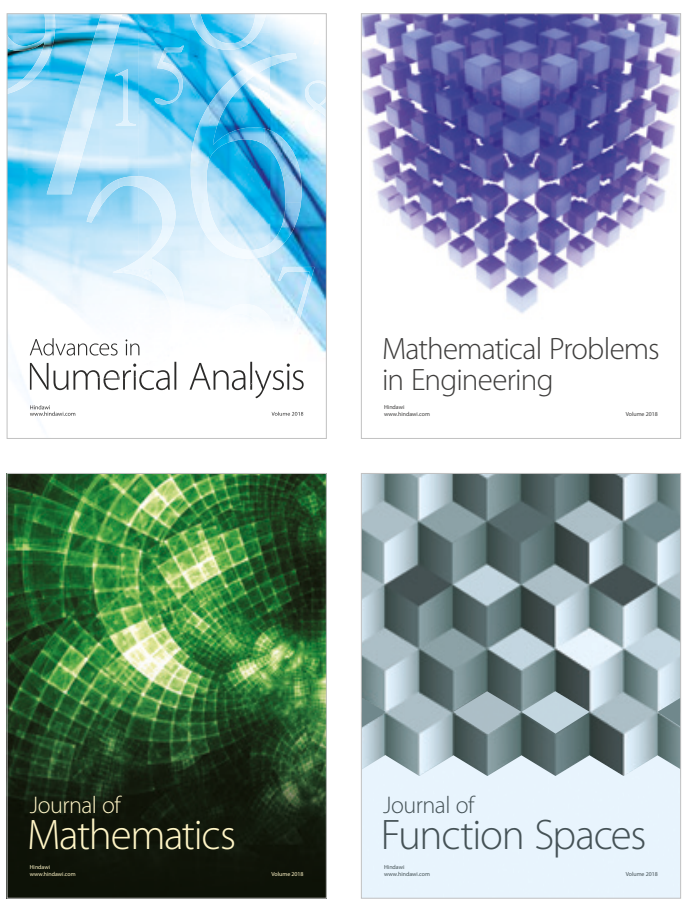

Mathematical Problems in Engineering

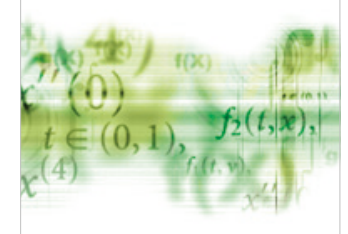

International Journal of

Differential Equations

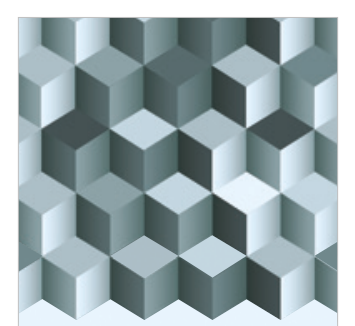

Journal of

Function Spaces
The Scientific

World Journal

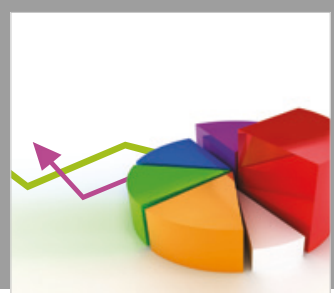

Journal of

Probability and Statistics
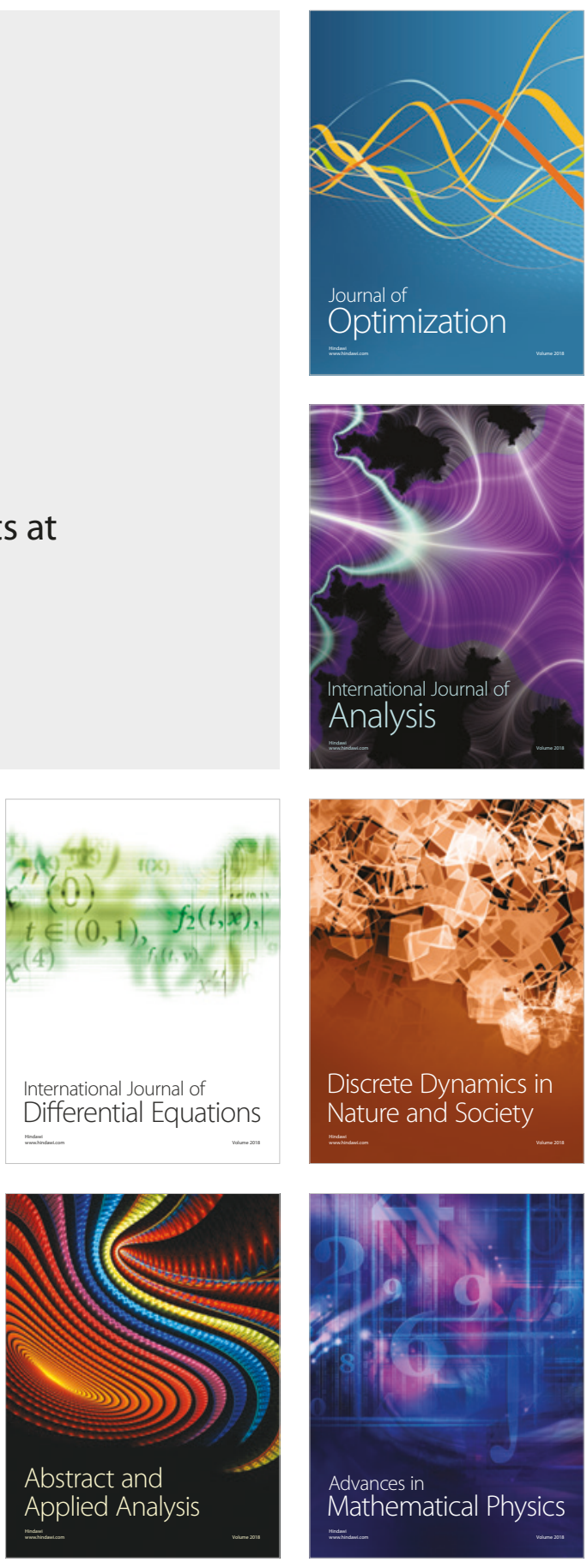